\title{
EXISTENCE AND BOUNDEDNESS OF PARAMETRIZED MARCINKIEWICZ INTEGRAL WITH ROUGH KERNEL ON CAMPANATO SPACES
}

\author{
YONG DING, QINGYING XUE AND KÔZÔ YABUTA
}

\begin{abstract}
Let $g(f), S(f), g_{\lambda}^{*}(f)$ be the Littlewood-Paley $g$ function, Lusin area function, and Littlewood-Paley $g_{\lambda}^{*}$ function of $f$, respectively. In 1990 Chen Jiecheng and Wang Silei showed that if, for a BMO function $f$, one of the above functions is finite for a single point in $\mathbb{R}^{n}$, then it is finite a.e. on $\mathbb{R}^{n}$, and BMO boundedness holds. Recently, Sun Yongzhong extended this result to the case of Campanato spaces (i.e. Morrey spaces, BMO, and Lipschitz spaces). One of us improved his $g_{\lambda}^{*}$ result further, and treated parametrized Marcinkiewicz functions with Lipschitz kernel $\mu^{\rho}(f), \mu_{S}^{\rho}(f)$ and $\mu_{\lambda}^{*, \rho}(f)$. In this paper, we show that the same results hold also in the case of rough kernel satisfying $L^{p}$-Dini type condition.
\end{abstract}

\section{$\S 1$. Introduction}

In this note we study the existence and boundedness property of parametrized Marcinkiewicz functions with rough kernel, on Campanato spaces. First, we recall the definition of Littlewood-Paley's functions (generalized ones) in the $n$-dimensional Euclidean space $\mathbb{R}^{n}(n \geq 2)$.

Let $\psi$ be a function $\psi$ on $\mathbb{R}^{n}$ such that there exist positive constants $C_{0}, C_{1}, \delta, \eta$ and $\gamma$ satisfying

(i) $\psi \in L^{1}\left(\mathbb{R}^{n}\right)$ and $\int_{\mathbb{R}^{n}} \psi(x) d x=0$;

(ii) $|\psi(x)| \leq C_{0}(1+|x|)^{-n-\delta}$;

(iii) $|\psi(x+h)-\psi(x)| \leq C_{1}|h|^{\gamma}(1+|x|)^{-n-\eta}$ for $|h| \leq|x| / 2$.

For this $\psi$, we define Littlewood-Paley's $g$, Lusin's area functions and Littlewood-Paley's $g_{\lambda}^{*}$ function as follows. Here and hereafter, $\psi_{t}(x)$ de-

Received October 1, 2004.

Revised April 7, 2005.

2000 Mathematics Subject Classification: 42B25.

The first named author was supported by NSF of China (Grant No. 10571015) and DPFIHE of China (Grant No. 20050027025). 
notes $t^{-n} \psi(x / t)$.

$$
\begin{gathered}
g(f)(x)=\left(\int_{0}^{\infty} \frac{\left|\psi_{t} * f(x)\right|^{2}}{t} d t\right)^{1 / 2}, \\
S(f)(x)=\left(\int_{\Gamma(x)}\left|\psi_{t} * f(y)\right|^{2} \frac{d y d t}{t^{n+1}}\right)^{1 / 2},
\end{gathered}
$$

where $\Gamma(x)=\left\{(y, t) \in \mathbb{R}_{+}^{n+1} ;|x-y|<t\right\}$.

$$
g_{\lambda}^{*}(f)(x)=\left(\int_{\mathbb{R}_{+}^{n+1}}\left(\frac{t}{t+|x-y|}\right)^{\lambda n}\left|\psi_{t} * f(y)\right|^{2} \frac{d y d t}{t^{n+1}}\right)^{1 / 2}
$$

where $\lambda>1 . L^{p}$ boundedness of these operators are known like as the classical Littlewood-Paley's $g$-functions. That is, $g$ and $S$ are $L^{p}$ bounded for $1<p<\infty$, and $g_{\lambda}^{*}$ is $L^{p}$ bounded for $1<p<\infty$ if $\lambda>\max \{1,2 / p\}$ (see for example Torchinsky [21, pp. 309-318]). Here and hereafter, the letter $C$ denotes a constant depending on main parameters and may vary at each occurrence.

Stein's generalization of the Marcinkiewicz function is as follows [17]: Let $\Omega(x)$ be a function on $\mathbb{R}^{n}$ which satisfies the following two conditions:

(i) $\Omega(x)$ is homogeneous of degree zero and continuous on the unit sphere $S^{n-1}$, and satisfies for some $0<\beta \leq 1$

$$
\left|\Omega\left(x^{\prime}\right)-\Omega\left(y^{\prime}\right)\right| \leq C\left|x^{\prime}-y^{\prime}\right|^{\beta}, \quad x^{\prime}, y^{\prime} \in S^{n-1} .
$$

(ii) $\int_{S^{n-1}} \Omega\left(x^{\prime}\right) d \sigma\left(x^{\prime}\right)=0$, where $d \sigma$ is the surface Lebesgue measure on $S^{n-1}$.

Define $\mu(f)(x)$ by

$$
\mu(f)(x)=\left(\int_{0}^{\infty} \frac{\left|\psi_{t} * f(x)\right|^{2}}{t} d t\right)^{1 / 2},
$$

where $\psi(x)=\frac{\Omega(x)}{|x|^{n-1}} \chi_{\{|x| \leq 1\}}$.

In their work on Marcinkiewicz integral, A. Torchinsky and S. Wang [22] introduced the Marcinkiewicz functions $\mu_{S}(f)$ and $\mu_{\lambda}^{*}(f)$ corresponding to the $S$ function and $g_{\lambda}^{*}$ function. They gave $L^{p}$ boundedness of $\mu_{S}(f)$ and $\mu_{\lambda}^{*}(f)$ for $p \geq 2$. On the other hand, in the connection of $\mu(f)$ a parametrized Marcinkiewicz function $\mu^{\rho}(f)$ was considered by L. Hörmander 
[11]. It corresponds to the case $\psi(x)=\Omega(x)|x|^{\rho-n} \chi_{\{|x| \leq 1\}}$. Thus, Sakamoto and Yabuta have considered in [28] parametrized $\mu_{S}^{\rho}(f)$ and $\mu_{\lambda}^{*, \rho}(f)$, where $\psi(x)=\Omega(x)|x|^{\rho-n} \chi_{\{|x| \leq 1\}}$, for $\rho \in \mathbb{C}$ with $\operatorname{Re} \rho=\sigma>0$. Introducing these parametrized operators and using a Hilbert space valued version of the complex interpolation theorem of analytic families of operators, they could show that in the case $n \geq 3, \mu_{S}(f)$ and $\mu_{\lambda}^{*}(f)$ are $L^{p}$ bounded for $p>2 n /(n+2)$ and $\lambda>\max \{1,2 / p\}$, respectively. $L^{p}$ boundedness for parametrized operators $\mu_{S}^{\rho}(f)$ and $\mu_{\lambda}^{*, \rho}(f)$ are well discussed in [16], [28], and further developed by many authors. Recently we have shown the $L^{p}$ boundedness under weaker condition on kernels than Lipschitz smoothness. To state it, we introduce the following smoothness for kernels. Let $\omega_{q}(\delta)$ be the $L^{q}$ modulus of continuity of $\Omega(1 \leq q<\infty)$, defined by

$$
\omega_{q}(\delta)=\sup _{|\gamma| \leq \delta}\left(\int_{S^{n-1}}\left|\Omega\left(\gamma x^{\prime}\right)-\Omega\left(x^{\prime}\right)\right|^{q} d \sigma\left(x^{\prime}\right)\right)^{1 / q},
$$

where $\gamma$ is a rotation on $S^{n-1}$, and $|\gamma|=\sup _{x^{\prime} \in S^{n-1}}\left|\gamma x^{\prime}-x^{\prime}\right|$. If $\omega_{q}(\delta)$ satisfies

$$
\int_{0}^{1} \frac{\omega_{q}(\delta)}{\delta} d \delta<\infty
$$

we say that $\Omega$ satisfies $L^{q}$-Dini condition. If $\omega_{q}(\delta)$ satisfies

$$
\int_{0}^{1} \frac{\omega_{q}(\delta)}{\delta}(1+|\log \delta|)^{\beta} d \delta<\infty
$$

we say that $\Omega$ satisfies $L^{q}-\log \beta$-Dini condition. If $\omega_{q}(\delta)$ satisfies

$$
\int_{0}^{1} \frac{\omega_{q}(\delta)}{\delta^{1+\beta}} d \delta<\infty
$$

we say that $\Omega$ satisfies $L^{q}-\beta$-Dini condition.

$L^{p}$ boudedness results are as follows.

Theorem A. (i) Let $\sigma>0, \max \left\{1, \frac{2 n}{n+2 \sigma}\right\}<p<2$, and $\lambda>2 / p$. Let $\Omega \in L^{2}\left(S^{n-1}\right)$ satisfy the cancellation condition $\int_{S^{n-1}} \Omega\left(x^{\prime}\right) d \sigma\left(x^{\prime}\right)=0$ and $L^{2}-\log \beta$-Dini condition for some $\beta>1$. Then, there exist $C_{1}, C_{2}>0$, independent of $f$, such that

$$
\left\|\mu_{S}^{\rho}(f)\right\|_{p} \leq C_{1}\left\|\mu_{\lambda}^{*, \rho}(f)\right\|_{p} \leq C_{2}\|f\|_{p} .
$$


(ii) Let $\sigma>0,2 \leq p<\infty$, and $\lambda>1$. Let $\Omega \in L \log ^{+} L\left(S^{n-1}\right)$ satisfy the cancellation condition $\int_{S^{n-1}} \Omega\left(x^{\prime}\right) d \sigma\left(x^{\prime}\right)=0$. Then, there exist $C_{1}$, $C_{2}>0$, independent of $f$, such that

$$
\left\|\mu_{S}^{\rho}(f)\right\|_{p} \leq C_{1}\left\|\mu_{\lambda}^{*, \rho}(f)\right\|_{p} \leq C_{2}\|f\|_{p}
$$

(See [8] for the proof of Theorem A (i) and [6] for the proof of Theo$\operatorname{rem} \mathrm{A}(\mathrm{ii})$.

As for $\mu^{\rho}$, we have

Theorem B. Let $\sigma>0,1<p<\infty$. Let $\Omega \in H^{1}\left(S^{n-1}\right)$ (the Hardy space on $\left.S^{n-1}\right)$ satisfy the cancellation condition $\int_{S^{n-1}} \Omega\left(x^{\prime}\right) d \sigma\left(x^{\prime}\right)=0$. Then, there exists $C>0$, independent of $f$, such that

$$
\left\|\mu^{\rho}(f)\right\|_{p} \leq C\|f\|_{p}
$$

(See [4] for the proof of Theorem B in the case $\rho \equiv 1$. The proof of Theorem B for $\rho \in \mathbb{C}$ with $\sigma>0$ can be obtained by the same way as in [4].) We note the following:

$$
\begin{aligned}
\operatorname{Lip}_{\alpha}\left(S^{n-1}\right)(0<\alpha \leq 1) & \varsubsetneqq L^{q}\left(S^{n-1}\right)(q>1) \varsubsetneqq L \log ^{+} L\left(S^{n-1}\right) \\
& \varsubsetneqq H^{1}\left(S^{n-1}\right) .
\end{aligned}
$$

It is also known that, if $\Omega \in L^{1}\left(S^{n-1}\right)$ satisfies $L^{1}$-Dini condition, then $\Omega \in L \log ^{+} L\left(S^{n-1}\right)$, see [1].

We recall also the definition of Campanato spaces [14].

Definition 1.1. For $1 \leq p<\infty$ and $-n / p \leq \alpha \leq 1$, the Campanato space $\mathcal{E}^{\alpha, p}$ is defined by the set of functions for which

$$
\|f\|_{\mathcal{E}^{\alpha, p}}=\sup _{x_{0} \in \mathbb{R}^{n}} \sup _{B} \frac{1}{|B|^{\alpha / n}}\left(\frac{1}{|B|} \int_{B}\left|f(x)-f_{B}\right|^{p} d x\right)^{1 / p}<\infty,
$$

where $B$ moves over all balls centered at $x_{0}$, and $f_{B}$ is the average of $f$ over $B,(1 /|B|) \int_{B} f(t) d t$.

It is known that for $0<\alpha \leq 1, \mathcal{E}^{\alpha, p}=\operatorname{Lip}_{\alpha}$ : the Banach space of Lipschitz continuous functions of exponent $\alpha$, and the norms are equivalent. If $\alpha=0, \mathcal{E}^{\alpha, p}$ coincides with BMO: the space of functions of bounded mean oscillation. And if $\alpha<0, \mathcal{E}^{\alpha, p}$ is equivalent to the Morrey space $L^{p, n+p \alpha}$. It is also easily checked that $\|f\|_{\alpha, p} \leq C \sup _{B} \inf _{a \in \mathbb{C}}|B|^{-\alpha / n}\left(|B|^{-1} \int_{B} \mid f(x)-\right.$ 
$\left.\left.a\right|^{p} d x\right)^{1 / p}(-n / p \leq \alpha \leq 1)$, and hence these norms are equivalent. We note that balls can be replaced by cubes with sides parallel to the coordinate axes and the norms are equivalent.

In 1984, Wang Silei [24] showed that the BMO boundedness of Littlewood-Paley's $g$-function follows from its finiteness on a set of positive measure. Since then, many authors considered such problems in BMO, Lipschitz spaces, and Morrey spaces i.e. in Campanato spaces. In 1990, Wang Silei and Chen Jiecheng [25] showed that the BMO boundedness follows from its finiteness at only one point for Littlewood-Paley's $g$-function, Lusin's area function and Littlewood-Paley's $g^{*}$-function, and Marcinkiewicz function. Recently, Sun Yongzhong [20] improves and extends their results to the case of Campanato spaces. Further, one of us [29] improves Sun's result and also treats the case of parametrized Marcinkiewicz integrals. In this paper, we improve the results in [29], i.e. we treat parametrized Marcinkiewicz integrals with more rough kernels.

Our results are as follows, where $\rho \in \mathbb{C}$ and $\operatorname{Re} \rho=\sigma$.

Theorem 1. Let $\sigma>0$, and suppose that $\Omega \in L^{q}\left(S^{n-1}\right)$ and satisfies $L^{q}-\log 1-D i n i$ condition for some $q>1$ and the cancellation condition. Then, if $f \in \mathrm{BMO}\left(\mathbb{R}^{n}\right)$ and $\mu^{\rho}(f)(x)$ is finite for a point $x_{0} \in \mathbb{R}^{n}$, it follows $\mu^{\rho}(f)(x)<\infty$ a.e. on $\mathbb{R}^{n}$, and there is a constant $C$ independent of $f$, such that

$$
\left\|\mu^{\rho}(f)\right\|_{\mathrm{BMO}\left(\mathbb{R}^{n}\right)} \leq C\|f\|_{\mathrm{BMO}\left(\mathbb{R}^{n}\right)} .
$$

Theorem 2. Let $\sigma>0$, and suppose that $\Omega \in L^{1}\left(S^{n-1}\right)$ and satisfies $L^{1}-\beta$-Dini condition for some $0<\beta \leq 1$ and the cancellation condition. Then, if $f \in \operatorname{Lip}_{\alpha}\left(\mathbb{R}^{n}\right)$ for $0<\alpha<\beta$ and $\mu^{\rho}(f)(x)$ is finite for a point $x_{0} \in \mathbb{R}^{n}$, it follows $\mu^{\rho}(f)(x)<\infty$ a.e. on $\mathbb{R}^{n}$, and there is a constant $C$ independent of $f$, such that

$$
\left\|\mu^{\rho}(f)\right\|_{\operatorname{Lip}_{\alpha}\left(\mathbb{R}^{n}\right)} \leq C\|f\|_{\operatorname{Lip}_{\alpha}\left(\mathbb{R}^{n}\right)} .
$$

Theorem 3. Let $\sigma>0,1<p<\infty$ and $-n / p \leq \alpha<0$. Moreover, suppose $\Omega \in L^{p^{\prime}}\left(S^{n-1}\right)$ and satisfies $L^{p^{\prime}}$-Dini condition and the cancellation condition. Then, if $f \in \mathcal{E}^{\alpha, p}$ and $\mu^{\rho}(f)(x)$ is finite for a point $x_{0} \in \mathbb{R}^{n}$, it follows $\mu^{\rho}(f)(x)<\infty$ a.e. on $\mathbb{R}^{n}$, and there is a constant $C$ independent of $f$, such that

$$
\left\|\mu^{\rho}(f)\right\|_{\mathcal{E}^{\alpha, p}} \leq C\|f\|_{\mathcal{E}^{\alpha, p}}
$$


THEOREM 4. Let $\sigma>0$, and suppose that $\Omega \in L^{q}\left(S^{n-1}\right)$ for some $q>1$ and satisfies the cancellation condition. Then, if $f \in \operatorname{BMO}\left(\mathbb{R}^{n}\right)$ and $\mu_{S}^{\rho}(f)(x)$ is finite for a point $x_{0} \in \mathbb{R}^{n}$, it follows $\mu_{S}^{\rho}(f)(x)<\infty$ a.e. on $\mathbb{R}^{n}$, and there is a constant $C$ independent of $f$, such that

$$
\left\|\mu_{S}^{\rho}(f)\right\|_{\mathrm{BMO}\left(\mathbb{R}^{n}\right)} \leq C\|f\|_{\mathrm{BMO}\left(\mathbb{R}^{n}\right)} .
$$

TheOREM 5. Let $\sigma>0,0<\alpha<1$, and suppose that $\Omega \in$ $L \log ^{+} L\left(S^{n-1}\right)$ and satisfies the cancellation condition if $0<\alpha<1 / 2$, and $\Omega \in L^{1}\left(S^{n-1}\right)$ and satisfies $L^{1}-\beta$-Dini condition for some $0<\beta \leq 1$ and the cancellation condition if $1 / 2 \leq \alpha<1$. Then, if $f \in \operatorname{Lip}_{\alpha}\left(\mathbb{R}^{n}\right)$ for $0<\alpha<1 / 2$ or $1 / 2 \leq \alpha<\min \{\beta, \sigma\}$ and $\mu_{S}^{\rho}(f)(x)$ is finite for a point $x_{0} \in \mathbb{R}^{n}$, it follows $\mu_{S}^{\rho}(f)(x)<\infty$ a.e. on $\mathbb{R}^{n}$, and there is a constant $C$ independent of $f$, such that

$$
\left\|\mu_{S}^{\rho}(f)\right\|_{\operatorname{Lip}_{\alpha}\left(\mathbb{R}^{n}\right)} \leq C\|f\|_{\operatorname{Lip}_{\alpha}\left(\mathbb{R}^{n}\right)} .
$$

Theorem 6. Let $1<p<\infty,-n / p \leq \alpha<0$. Suppose the number $\sigma$ and the kernel $\Omega$ satisfy one of the following conditions:

(i) $\sigma>-\alpha, \max \left\{1, \frac{2 n}{n+2 \sigma}\right\}<p, \Omega \in L^{\max \left\{2, p^{\prime}\right\}}\left(S^{n-1}\right)$ and $\Omega$ satisfies the cancellation condition. In the case $p<2, \Omega$ moreover satisfies $L^{2}-\log \beta$ Dini condition for some $\beta>1$.

(ii) $\sigma>n / 2, \Omega \in L^{2}\left(S^{n-1}\right)$ and $\Omega$ satisfies $L^{2}-\log \beta$-Dini condition for some $\beta>1$ and the cancellation condition.

Then, if $f \in \mathcal{E}^{\alpha, p}$ and $\mu_{S}^{\rho}(f)(x)$ is finite for a point $x_{0} \in \mathbb{R}^{n}$, it follows $\mu_{S}^{\rho}(f)(x)<\infty$ a.e. on $\mathbb{R}^{n}$, and there is a constant $C$ independent of $f$, such that

$$
\left\|\mu_{S}^{\rho}(f)\right\|_{\mathcal{E}^{\alpha, p}} \leq C\|f\|_{\mathcal{E}^{\alpha, p}} .
$$

THEOREM 7. Let $\sigma>0, \lambda>1$, and suppose that $\Omega \in L^{q}\left(S^{n-1}\right)$ for some $q>1$ and satisfies the cancellation condition. Then, if $f \in \operatorname{BMO}\left(\mathbb{R}^{n}\right)$ and $\mu_{\lambda}^{*, \rho}(f)(x)$ is finite for a point $x_{0} \in \mathbb{R}^{n}$, it follows $\mu_{\lambda}^{*, \rho}(f)(x)<\infty$ a.e. on $\mathbb{R}^{n}$, and there is a constant $C$ independent of $f$, such that

$$
\left\|\mu_{\lambda}^{*, \rho}(f)\right\|_{\mathrm{BMO}\left(\mathbb{R}^{n}\right)} \leq C\|f\|_{\mathrm{BMO}\left(\mathbb{R}^{n}\right)} .
$$

TheOREM 8. Let $\sigma>0,0<\alpha<1$, and suppose that $\Omega \in$ $L \log ^{+} L\left(S^{n-1}\right)$ and satisfies the cancellation condition if $0<\alpha<1 / 2$, and $\Omega \in L^{1}\left(S^{n-1}\right)$ and satisfies $L^{1}-\beta$-Dini condition for some $0<\beta \leq 1$ 
and the cancellation condition if $1 / 2 \leq \alpha<1$. Suppose that $f \in \operatorname{Lip}_{\alpha}\left(\mathbb{R}^{n}\right)$ for $0<\alpha<1 / 2$ or $1 / 2 \leq \alpha<\min \{\beta, \sigma\}$ and $\mu_{\lambda}^{*, \rho}(f)(x)$ is finite for a point $x_{0} \in \mathbb{R}^{n}, \lambda>\lambda_{0}$, where $\lambda_{0}=1$ for $0<\alpha<1 / 2$, and $\lambda_{0}=1+2 \alpha / n$ for $1 / 2 \leq \alpha<1$. Then $\mu_{\lambda}^{*, \rho}(f)(x)<\infty$ a.e. on $\mathbb{R}^{n}$, and there is a constant $C$ independent of $f$, such that

$$
\left\|\mu_{\lambda}^{*, \rho}(f)\right\|_{\operatorname{Lip}_{\alpha}\left(\mathbb{R}^{n}\right)} \leq C\|f\|_{\operatorname{Lip}_{\alpha}\left(\mathbb{R}^{n}\right)} .
$$

TheOREM 9. Let $1<p<\infty,-n / p \leq \alpha<0$. Suppose the positive numbers $\sigma, \lambda$ and the kernel $\Omega$ satisfy one of the following conditions:

(i) $\sigma>-\alpha, \max \left\{1, \frac{2 n}{n+2 \sigma}\right\}<p, \lambda>\max \{1,2 / p\}, \Omega \in L^{\max \left\{2, p^{\prime}\right\}}\left(S^{n-1}\right)$ and $\Omega$ satisfies the cancellation condition. In the case $p<2, \Omega$ moreover satisfies $L^{2}-\log \beta$-Dini condition for some $\beta>1$.

(ii) $\sigma>n / 2, \lambda>2, \Omega \in L^{2}\left(S^{n-1}\right)$ and $\Omega$ satisfies $L^{2}-\log \beta$-Dini condition for some $\beta>1$ and the cancellation condition.

Then, if $f \in \mathcal{E}^{\alpha, p}$ and $\mu_{\lambda}^{*, \rho}(f)(x)$ is finite for a point $x_{0} \in \mathbb{R}^{n}$, it follows $\mu_{\lambda}^{*, \rho}(f)(x)<\infty$ a.e. on $\mathbb{R}^{n}$, and there is a constant $C$ independent of $f$, such that

$$
\left\|\mu_{\lambda}^{*, \rho}(f)\right\|_{\mathcal{E}^{\alpha, p}} \leq C\|f\|_{\mathcal{E}^{\alpha, p}} .
$$

To prove the above theorems we use the following key lemmas.

Lemma 1.1. Let $1 \leq p<\infty$. If $\delta>0$ and $-n / p \leq \alpha<\min \{1, \delta / p\}$, then there exists $C>0$ such that for any ball $B=B(x, r)$ and any $f \in \mathcal{E}^{\alpha, p}$

$$
\left(\int_{\mathbb{R}^{n}} \frac{\left|f(y)-f_{B}\right|^{p}}{(r+|y-x|)^{n+\delta}} d y\right)^{1 / p} \leq C r^{\alpha-\delta / p}\|f\|_{\mathcal{E}^{\alpha, p}} .
$$

This can be proved easily by modifying the proof of Lemma 2.3 in [9]. We need also the following lemma, which is an extension of the result obtained by Kurtz and Wheeden in 1979 [13], and whose proof is found in [8].

LemmA 1.2. Let $1 \leq q<\infty$ and $\rho=\sigma+i \tau(\sigma, \tau \in \mathbb{R})$ with $\sigma>0$. Suppose that $\Omega$ is homogeneous of degree zero and satisfies the $L^{q}$-Dini condition. Then, there exists $C>0$ such that for any $R>0$ and $|y|<\frac{1}{2} R$,

$$
\begin{aligned}
& \left(\int_{R<|x|<2 R}\left|\frac{\Omega(x-y)}{|x-y|^{n-\rho}}-\frac{\Omega(x)}{|x|^{n-\rho}}\right|^{q} d x\right)^{1 / q} \\
& \leq C(1+|\tau|) R^{n / q-(n-\sigma)}\left\{\|\Omega\|_{L^{q}\left(S^{n-1}\right)} \frac{|y|}{R}+\int_{|y| / 2 R}^{|y| / R} \frac{\omega_{q}(\delta)}{\delta} d \delta\right\},
\end{aligned}
$$


where $C$ is independent of $R$ and $y$.

We shall use the following elementary lemma, whose proof we omit.

Lemma 1.3. (1) Let $\sigma>0$. Then there exists $C>0$ such that

$$
\int_{t-r}^{t} s^{\sigma-1} d s \leq C r t^{\sigma-1}, \quad 0<r<t .
$$

(2) Let $\sigma \leq 0$ and $a>1$. Then there exists $C>0$ such that

$$
\int_{t-r}^{t} s^{\sigma-1} d s \leq C r t^{\sigma-1}, \quad 0<a r<t .
$$

In the next section, we prepare several lemmas to prove Theorems 1-9, and in Section 3, we shall prove them. Lemmas 2.1, 2.2, 2.3 are for the proofs of Theorems 1, 2, 3. Lemmas 2.4, 2.5, 2.6 are for the proofs of Theorems 4, 5, 6. And Lemmas 2.7, 2.8, 2.9, 2.10, 2.11, 2.12 are for the proofs of Theorems $7,8,9$.

\section{§2. Lemmas}

In this section, $r$ is a temporarily fixed positive number. For a ball $B=B\left(x_{0}, r\right)$ and a function $f$ we set always $f_{1}=f_{4 B}, f_{2}=\left(f(y)-f_{4 B}\right) \chi_{4 B}$ and $f_{3}=\left(f(y)-f_{4 B}\right) \chi_{(4 B)^{c}}$. To proceed as in the proof of Theorem 3 in Yabuta [29], we introduce auxiliary operators depending on $r$ as follows. Relating to $\mu^{\rho}(f)$, we define the following.

DEFINITION 2.1.

$$
\mu_{0}^{\rho}(f)(x)=\left(\int_{0}^{r}\left|\frac{1}{t^{\rho}} \int_{|y-x| \leq t} \frac{\Omega(y-x)}{|y-x|^{n-\rho}} f(y) d y\right|^{2} \frac{d t}{t}\right)^{1 / 2}
$$

and

$$
\mu_{\infty}^{\rho}(f)(x)=\left(\int_{r}^{\infty}\left|\frac{1}{t^{\rho}} \int_{|y-x| \leq t} \frac{\Omega(y-x)}{|y-x|^{n-\rho}} f(y) d y\right|^{2} \frac{d t}{t}\right)^{1 / 2} .
$$

Relating to $\mu_{S}^{\rho}(f)$, we define the following.

DEFINITION 2.2.

$$
\mu_{S, 0}^{\rho}(f)(x)=\left(\int_{0}^{r} \int_{|u-x| \leq t}\left|\frac{1}{t^{\rho}} \int_{|y-u| \leq t} \frac{\Omega(u-y) f(y)}{|u-y|^{n-\rho}} d y\right|^{2} \frac{d u d t}{t^{n+1}}\right)^{1 / 2},
$$


and

$$
\mu_{S, \infty}^{\rho}(f)(x)=\left(\int_{r}^{\infty} \int_{|u-x| \leq t}\left|\frac{1}{t^{\rho}} \int_{|y-u| \leq t} \frac{\Omega(u-y) f(y)}{|u-y|^{n-\rho}} d y\right|^{2} \frac{d u d t}{t^{n+1}}\right)^{1 / 2} .
$$

Relating to $\mu_{\lambda}^{*, \rho}(f)$, we define the following.

\section{DEFINITION 2.3.}

$$
\begin{aligned}
& \mu_{\lambda, 0}^{*, \rho}(f)(x) \\
& =\left(\int_{0}^{r} \int_{\mathbb{R}^{n}}\left|\frac{1}{t^{\rho}} \int_{|y-u| \leq t} \frac{\Omega(u-y) f(y)}{|u-y|^{n-\rho}} d y\right|^{2}\left(\frac{t}{t+|u-x|}\right)^{\lambda n} \frac{d u d t}{t^{n+1}}\right)^{1 / 2}, \\
& \mu_{\lambda, \infty}^{*, \rho}(f)(x) \\
& =\left(\int_{r}^{\infty} \int_{\mathbb{R}^{n}}\left|\frac{1}{t^{\rho}} \int_{|y-u| \leq t} \frac{\Omega(u-y) f(y)}{|u-y|^{n-\rho}} d y\right|^{2}\left(\frac{t}{t+|u-x|}\right)^{\lambda n} \frac{d u d t}{t^{n+1}}\right)^{1 / 2}, \\
& \mu_{\lambda, 0,0}^{*, \rho}(f)(x) \\
& =\left(\int_{0}^{r} \int_{|u-x| \leq 8 r}\left|\frac{1}{t^{\rho}} \int_{|y-u| \leq t} \frac{\Omega(u-y) f(y)}{|u-y|^{n-\rho}} d y\right|^{2}\left(\frac{t}{t+|u-x|}\right)^{\lambda n} \frac{d u d t}{t^{n+1}}\right)^{1 / 2},
\end{aligned}
$$

and

$$
\begin{aligned}
& \mu_{\lambda, 0, \infty}^{*, \rho}(f)(x) \\
& =\left(\int_{0}^{r} \int_{|u-x|>8 r}\left|\frac{1}{t^{\rho}} \int_{|y-u| \leq t} \frac{\Omega(u-y) f(y)}{|u-y|^{n-\rho}} d y\right|^{2}\left(\frac{t}{t+|u-x|}\right)^{\lambda n} \frac{d u d t}{t^{n+1}}\right)^{1 / 2} .
\end{aligned}
$$

First, we prepare three lemmas to prove Theorems 1, 2, and 3.

LEMMA 2.1. Let $1 \leq p<\infty$. Let $\Omega \in L^{p^{\prime}}\left(S^{n-1}\right), \int_{S^{n-1}} \Omega(x) d \sigma(x)=$ $0,-n / p \leq \alpha<1$, and $\rho=\sigma+i \tau(\sigma>0, \tau \in \mathbb{R})$. Then, if $f \in \mathcal{E}^{\alpha, p}$ and $\mu^{\rho}(f)\left(x_{0}\right)<+\infty$ for some $x_{0} \in \mathbb{R}^{n}$, there exists $C>0$ such that for any ball $B=B\left(x_{0}, r\right)$

$$
\mu_{\infty}^{\rho}\left(f_{2}\right)\left(x_{0}\right) \leq C\left(\mu^{\rho}(f)\left(x_{0}\right)+\|\Omega\|_{L^{p^{\prime}}\left(S^{n-1}\right)} r^{\alpha}\|f\|_{\left.\mathcal{E}^{\alpha, p}\right)} .\right.
$$

In the case $0<\alpha<1, \Omega \in L^{p^{\prime}}\left(S^{n-1}\right)$ and $\|\Omega\|_{L^{p^{\prime}}\left(S^{n-1}\right)}$ can be replaced by $\Omega \in L^{1}\left(S^{n-1}\right)$ and $\|\Omega\|_{L^{1}\left(S^{n-1}\right)}$. 
Proof. By assumption we have

$$
\left(\int_{r}^{2 r}\left|\frac{1}{t^{\rho}} \int_{\left|y-x_{0}\right| \leq t} \frac{\Omega\left(y-x_{0}\right)}{\left|y-x_{0}\right|^{n-\rho}} f(y) d y\right|^{2} \frac{d t}{t}\right)^{1 / 2} \leq \mu^{\rho}(f)\left(x_{0}\right)<+\infty .
$$

Hence, for some $r \leq t_{0} \leq 2 r$ we get

$$
\frac{r}{t_{0}}\left|\frac{1}{t_{0}^{\rho}} \int_{\left|y-x_{0}\right| \leq t_{0}} \frac{\Omega\left(y-x_{0}\right)}{\left|y-x_{0}\right|^{n-\rho}} f(y) d y\right| \leq \mu^{\rho}(f)\left(x_{0}\right) .
$$

Since, in the above integral, the integration domain is contained in $\left|y-x_{0}\right| \leq$ $4 r$, we see, using the cancellation property of $\Omega$, that the above integral is equal to

$$
\int_{\left|y-x_{0}\right| \leq t_{0}} \frac{\Omega\left(y-x_{0}\right)}{\left|y-x_{0}\right|^{n-\rho}}\left(f(y)-f_{4 B}\right) \chi_{4 B} d y \text {. }
$$

Hence

$$
\left|\int_{\left|y-x_{0}\right| \leq t_{0}} \frac{\Omega\left(y-x_{0}\right)}{\left|y-x_{0}\right|^{n-\rho}}\left(f(y)-f_{4 B}\right) \chi_{4 B} d y\right| \leq C r^{\sigma} \mu^{\rho}(f)\left(x_{0}\right) .
$$

Thus for $t>r$ we have

$$
\begin{aligned}
& \left|\int_{\left|y-x_{0}\right| \leq t} \frac{\Omega\left(y-x_{0}\right)}{\left|y-x_{0}\right|^{n-\rho}} f_{2}(y) d y\right| \\
& \leq\left|\int_{\left|y-x_{0}\right| \leq t_{0}} \frac{\Omega\left(y-x_{0}\right)}{\left|y-x_{0}\right|^{n-\rho}} f_{2}(y) d y\right| \\
& \quad+\int_{t_{0}<\left|y-x_{0}\right|<\min \{t, 4 r\}} \frac{\left|\Omega\left(y-x_{0}\right)\right|\left|f(y)-f_{4 B}\right|}{\left|y-x_{0}\right|^{n-\sigma}} d y \\
& \leq C r^{\sigma} \mu^{\rho}(f)\left(x_{0}\right)+C r^{\sigma-n} \int_{\left|y-x_{0}\right|<4 r}\left|\Omega\left(y-x_{0}\right)\right|\left|f(y)-f_{4 B}\right| d y \\
& \leq C r^{\sigma} \mu^{\rho}(f)\left(x_{0}\right)+C r^{\sigma-n}\left(\int_{\left|y-x_{0}\right|<4 r}\left|\Omega\left(y-x_{0}\right)\right|^{p^{\prime}} d y\right)^{1 / p^{\prime}} \\
& \quad \times\left(\int_{\left|y-x_{0}\right|<4 r}\left|f(y)-f_{4 B}\right|^{p} d y\right)^{1 / p} \\
& \leq C r^{\sigma} \mu^{\rho}(f)\left(x_{0}\right) \\
& \quad+C r^{\sigma-n} r^{n / p^{\prime}}\|\Omega\|_{L^{p^{\prime}}\left(S^{n-1}\right)}\left(\int_{\left|y-x_{0}\right|<4 r}\left|f(y)-f_{4 B}\right|^{p} d y\right)^{1 / p} \\
& \leq C r^{\sigma} \mu^{\rho}(f)\left(x_{0}\right)+C\|\Omega\|_{L^{p^{\prime}}\left(S^{n-1}\right)} r^{\sigma+\alpha}\|f\|_{\mathcal{E}^{\alpha, p} .}
\end{aligned}
$$


Therefore we have

$$
\begin{aligned}
\mu_{\infty}^{\rho}\left(f_{2}\right)\left(x_{0}\right) & =\left(\int_{r}^{\infty}\left|\frac{1}{t^{\rho}} \int_{\left|y-x_{0}\right| \leq t} \frac{\Omega\left(y-x_{0}\right)}{\left|y-x_{0}\right|^{n-\rho}} f_{2}(y) d y\right|^{2} \frac{d t}{t}\right)^{1 / 2} \\
& \leq C r^{\sigma}\left(\mu^{\rho}(f)\left(x_{0}\right)+\|\Omega\|_{L^{p^{\prime}\left(S^{n-1}\right)}} r^{\alpha}\|f\|_{\mathcal{E}^{\alpha, p}}\right)\left(\int_{r}^{\infty} \frac{d t}{t^{2 \sigma+1}}\right)^{1 / 2} \\
& \leq C\left(\mu^{\rho}(f)\left(x_{0}\right)+\|\Omega\|_{L^{p^{\prime}\left(S^{n-1}\right)}} r^{\alpha}\|f\|_{\mathcal{E}^{\alpha, p}}\right) .
\end{aligned}
$$

In the case $0<\alpha<1$, for $\left|y-x_{0}\right|<4 r$ and $\left|z-x_{0}\right|<4 r$, we have $|y-z| \leq\left|y-x_{0}\right|+\left|x_{0}-z\right|<8 r$, and hence

$$
\begin{aligned}
\left|f(y)-f_{4 B}\right| & =\left|\frac{1}{|4 B|} \int_{4 B}(f(y)-f(z)) d z\right| \\
& \leq\|f\|_{\operatorname{Lip}_{\alpha}} \frac{1}{|4 B|} \int_{4 B}|y-z|^{\alpha} d z \leq C\|f\|_{\operatorname{Lip}_{\alpha}} r^{\alpha} .
\end{aligned}
$$

Thus,

$$
\begin{aligned}
& \int_{t_{0}<\left|y-x_{0}\right|<\min (t, 4 r)} \frac{\left|\Omega\left(y-x_{0}\right)\right|\left|f(y)-f_{4 B}\right|}{\left|y-x_{0}\right|^{n-\sigma}} d y \\
& \leq C\|f\|_{\operatorname{Lip}_{\alpha}} r^{\alpha} \int_{\left|y-x_{0}\right|<4 r} \frac{\left|\Omega\left(y-x_{0}\right)\right|}{\left|y-x_{0}\right|^{n-\sigma}} d y \\
& \leq C\|f\|_{\operatorname{Lip}_{\alpha}} r^{\alpha}\|\Omega\|_{L^{1}\left(S^{n-1}\right)} r^{\sigma} .
\end{aligned}
$$

Hence, pursueing the rest process in the above proof, we obtain the desired conclusion.

Lemma 2.2. Let $\Omega \in L^{1}\left(S^{n-1}\right)$ and $\rho \in \mathbb{C}$. Then, for any $f \in$ $L_{\text {loc }}^{1}\left(\mathbb{R}^{n}\right)$, for any ball $B=B\left(x_{0}, r\right)$ and any $x \in B$

$$
\mu_{0}^{\rho}\left(f_{3}\right)(x)=0
$$

Proof. For $\left|x-x_{0}\right| \leq r$ and $|x-y| \leq t \leq r$, we have $\left|x_{0}-y\right| \leq 2 r$, and hence the integration domain with respect to $y$ has no intersection with the support of $f_{3}$ in the expression of $\mu_{0}^{\rho}\left(f_{3}\right)$. So, we have $\mu_{0}^{\rho}\left(f_{3}\right)=0$ for $x \in B$.

LEMMA 2.3. Suppose one of the following three conditions is satisfied:

(i) $\sigma>0, \alpha=0, \Omega \in L^{q}\left(S^{n-1}\right)$ and $\Omega$ satisfies $L^{q}-\log 1-D i n i$ condition for some $q>1$ and the cancellation condition. 
(ii) $\sigma>0,0<\beta \leq 1,0<\alpha<\min \{\sigma, \beta\} \leq 1,1 \leq p<\infty$, $\Omega \in L^{1}\left(S^{n-1}\right)$ and $\Omega$ satisfies $L^{1}-\beta$-Dini condition and the cancellation condition.

(iii) $\sigma>0,1<p<\infty$ and $-n / p \leq \alpha<0$. Moreover, suppose $\Omega \in L^{p^{\prime}}\left(S^{n-1}\right)$ and satisfies $L^{p^{\prime}}$-Dini condition.

Then there exists $C>0$ such that for any ball $B=B\left(x_{0}, r\right)$ and any $f \in \mathcal{E}^{\alpha, p}$ satisfying $\mu_{\infty}^{\rho}\left(f_{3}\right)\left(x_{0}\right)<+\infty$, it holds

$$
\mu_{\infty}^{\rho}\left(f_{3}\right)(x)<+\infty \quad \text { and } \quad\left|\mu_{\infty}^{\rho}\left(f_{3}\right)(x)-\mu_{\infty}^{\rho}\left(f_{3}\right)\left(x_{0}\right)\right| \leq C r^{\alpha}\|f\|_{\mathcal{E}^{\alpha, p}}
$$

for any $x \in B$.

Proof. For any $x \in B$ we have

$$
\begin{aligned}
\left|\mu_{\infty}^{\rho}\left(f_{3}\right)(x)-\mu_{\infty}^{\rho}\left(f_{3}\right)\left(x_{0}\right)\right| & \left(\int_{r}^{\infty} \mid \frac{1}{t^{\rho}} \int_{|y-x|<t} \frac{\Omega(y-x) f_{3}(y) d y}{|y-x|^{n-\rho}}\right. \\
\leq & \left.\quad-\left.\frac{1}{t^{\rho}} \int_{\left|y-x_{0}\right|<t} \frac{\Omega\left(y-x_{0}\right) f_{3}(y) d y}{\left|y-x_{0}\right|^{n-\rho}}\right|^{2} \frac{d t}{t}\right)^{1 / 2} \\
\leq & \left(\int_{r}^{\infty}\left(\frac{1}{t^{\sigma}} \int_{|y-x|<t} \frac{\left|\Omega(y-x) f_{3}(y)\right| d y}{|y-x|^{n-\sigma}}\right)^{2} \frac{d t}{t}\right)^{1 / 2} \\
& +\left(\int_{r}^{\infty}\left(\frac{1}{t^{\sigma}} \int_{\substack{|y-x| \geq t \\
\left|y-x_{0}\right|<t}} \frac{\left|\Omega\left(y-x_{0}\right) f_{3}(y)\right| d y}{\left|y-x_{0}\right|^{n-\sigma}}\right)^{2} \frac{d t}{t}\right)^{1 / 2} \\
& +\left(\int_{r}^{\infty} \frac{1}{t^{2 \sigma}}\left(\int_{|y-x|<t}\left|\frac{\Omega(y-x)}{|y-x|^{n-\rho}}-\frac{\Omega\left(y-x_{0}\right)}{\left|y-x_{0}\right|^{n-\rho}}\right|_{\mid y t}\left|f_{3}(y)\right| d y\right)^{2} \frac{d t}{t}\right)^{1 / 2} \\
= & I_{1}(x)+I_{2}(x)+I_{3}(x) .
\end{aligned}
$$

(i) In this case, $\mathcal{E}^{0, p}=\mathrm{BMO}(1 \leq p<\infty)$, and the norms are equivalent. Take a positive constant $\eta$ with $0<\eta<q^{\prime}-1$. For $y \in(4 B)^{c},|y-x|<t$, $x \in B$, we have $t>\left|y-x_{0}\right|-\left|x-x_{0}\right|>3 r$. Noting this, we get by Hölder's inequality, Lemma 1.3 and Lemma 1.1

$$
\begin{aligned}
& \int_{\substack{|y-x|<t \\
\left|y-x_{0}\right| \geq t}} \frac{\left|\Omega(y-x) f_{3}(y)\right| d y}{|y-x|^{n-\sigma}} \\
& \leq\left(\int_{\max \{3 r, t-r\}<|y-x|<t} \frac{|\Omega(y-x)|^{q} d y}{|y-x|^{(n-\sigma)^{q}-(n+\eta) q / q^{\prime}}}\right)^{1 / q}\left(\int_{\mathbb{R}^{n}} \frac{\left|f_{3}(y)\right|^{q^{\prime}} d y}{|y-x|^{n+\eta}}\right)^{1 / q^{\prime}}
\end{aligned}
$$




$$
\begin{aligned}
& \leq\|\Omega\|_{L^{q}\left(S^{n-1}\right)}\left(\int_{\max \{3 r, t-r\}}^{t} s^{(n+\eta) q / q^{\prime}-(n-\sigma) q+n-1} d s\right)^{1 / q}\|f\|_{\mathrm{BMO}} r^{-\eta / q^{\prime}} \\
& \leq C\|\Omega\|_{L^{q}\left(S^{n-1}\right)}\|f\|_{\mathrm{BMO}} r^{-\eta / q^{\prime}} r^{1 / q} t^{\eta / q^{\prime}-1 / q+\sigma} .
\end{aligned}
$$

Thus, we have, noting $\eta / q^{\prime}-1 / q=\left(\eta-\left(q^{\prime}-1\right)\right) / q^{\prime}<0$,

$$
I_{1}(x) \leq C\|f\|_{\mathrm{BMO}} r^{-n / q^{\prime}+1 / q}\left(\int_{3 r}^{\infty} t^{2 \eta / q^{\prime}-2 / q-1} d t\right)^{1 / 2} \leq C\|f\|_{\mathrm{BMO}} .
$$

Similarly, we have the same estimate for $I_{2}(x)$.

As for $I_{3}(x)$, we have by Minkowski's inequality and Hölder's inequality

$$
\begin{aligned}
I_{3}(x) \leq & \int_{\mathbb{R}^{n}}\left|\frac{\Omega(y-x)}{|y-x|^{n-\rho}}-\frac{\Omega\left(y-x_{0}\right)}{\left|y-x_{0}\right|^{n-\rho}}\right|\left|f_{3}(y)\right|\left(\int_{r}^{\infty} \chi_{\left|y-x_{0}\right|<t} \frac{d t}{t^{2 \sigma+1}}\right)^{1 / 2} d y \\
\leq & C \int_{\left|y-x_{0}\right|>4 r}\left|\frac{\Omega(y-x)}{|y-x|^{n-\rho}}-\frac{\Omega\left(y-x_{0}\right)}{\left|y-x_{0}\right|^{n-\rho}}\right| \frac{\left|f_{3}(y)\right|}{\left|y-x_{0}\right|^{\sigma}} d y \\
= & \sum_{j=2}^{\infty} \int_{2^{j} r \leq\left|y-x_{0}\right|<2^{j+1} r} \mid \frac{\Omega(y-x)}{|y-x|^{n-\rho}}-\frac{\Omega\left(y-x_{0}\right)}{\left|y-x_{0}\right|^{n-\rho} \mid} \frac{\left|f_{3}(y)\right|}{\left|y-x_{0}\right|^{\sigma}} d y \\
\leq & \sum_{j=2}^{\infty}\left(\int_{2^{j} r \leq\left|y-x_{0}\right|<2^{j+1} r} \mid \frac{\Omega(y-x)}{|y-x|^{n-\rho}}-\frac{\Omega\left(y-x_{0}\right)}{\left.\left.\left|y-x_{0}\right|^{n-\rho}\right|^{q} d y\right)^{1 / q}}\right. \\
& \times\left(\int_{2^{j} r \leq\left|y-x_{0}\right|<2^{j+1} r} \frac{\left|f_{3}(y)\right|^{q^{\prime}}}{\left|y-x_{0}\right|^{q^{\prime} \sigma}} d y\right)^{1 / q^{\prime}} \cdot
\end{aligned}
$$

Now, since $\alpha=0$ and $f \in \mathrm{BMO}$, we see easily

$$
\begin{aligned}
& \left(\frac{1}{\left|2^{j+1} B\right|} \int_{2^{j} r \leq\left|y-x_{0}\right|<2^{j+1} r} \frac{\left|f_{3}(y)\right|^{q^{\prime}}}{\left|y-x_{0}\right|^{q^{\prime} \sigma}} d y\right)^{1 / q^{\prime}} \\
& \leq \frac{1}{\left(2^{j} r\right)^{\sigma}}\left(\frac{1}{\left|2^{j+1} B\right|} \int_{2^{j+1} B}\left|f(y)-f_{4 B}\right|^{q^{\prime}} d y\right)^{1 / q^{\prime}} \\
& \leq C \frac{\|f\|_{\mathrm{BMO}}}{\left(2^{j} r\right)^{\sigma}} j \leq C\|f\|_{\mathrm{BMO}} r^{-\sigma} 2^{-j \sigma} j .
\end{aligned}
$$

Hence, using Lemma 1.2, we get

$$
\begin{aligned}
I_{3}(x) \leq C \sum_{j=2}^{\infty} & \left(2^{j} r\right)^{n / q-(n-\sigma)}\left(\|\Omega\|_{L^{q}\left(S^{n-1}\right)} \frac{\left|x-x_{0}\right|}{2^{j} r}+\int_{\frac{\left|x-x_{0}\right|}{2^{j+1} r}}^{\frac{\left|x-x_{0}\right|}{2^{j} r}} \frac{\omega_{q}(\delta)}{\delta} d \delta\right) \\
& \times\|f\|_{\mathrm{BMO}} 2^{-j \sigma} r^{-\sigma}\left(2^{j} r\right)^{n / q^{\prime}} j
\end{aligned}
$$




$$
\begin{aligned}
& \leq C\|f\|_{\mathrm{BMO}} \sum_{j=2}^{\infty}\left(\|\Omega\|_{L^{q}\left(S^{n-1}\right)} 2^{-j} j+j \int_{\frac{\left|x-x_{0}\right|}{2^{j+1} r}}^{\frac{\left|x-x_{0}\right|}{2^{j} r}} \frac{\omega_{q}(\delta)}{\delta} d \delta\right) \\
& \leq C\|f\|_{\mathrm{BMO}}\left(\|\Omega\|_{L^{q}\left(S^{n-1}\right)}+\int_{0}^{1} \frac{\omega_{q}(\delta)}{\delta}(1+|\log \delta|) d \delta\right) .
\end{aligned}
$$

Summing up the estimates for $I_{1}(x), I_{2}(x), I_{3}(x)$, we obtain the desired estimate in the case (i).

(ii) In this case, $\mathcal{E}^{\alpha, p}=\operatorname{Lip}_{\alpha}(1 \leq p<\infty)$, and the norms are equivalent. For $\left|y-x_{0}\right| \geq 4 r$ and $\left|z-x_{0}\right|<4 r$, we have $|y-z| \leq|y-x|+\mid x-$ $x_{0}|+| x_{0}-z|\leq| y-x \mid+5 r$, and hence we have $\left|f_{3}(y)\right| \leq\left|f(y)-f_{4 B}\right| \leq$ $5 \cdot 2^{\alpha}\|f\|_{\operatorname{Lip}_{\alpha}}\left(|y-x|^{\alpha}+r^{\alpha}\right)$. Also, $|y-x|<t$ and $\left|y-x_{0}\right| \geq t>r$ implies $t-r \leq\left|y-x_{0}\right|-\left|x_{0}-x\right| \leq|x-y|<t$. So, for $I_{1}$ we have using Lemma 1.3

$$
\begin{aligned}
I_{1}(x) & \leq C\|f\|_{\operatorname{Lip}_{\alpha}}\left(\int_{r}^{\infty}\left(\frac{1}{t^{\sigma}} \int_{\substack{|y-x|<t \\
\left|y-x_{0}\right| \geq t}} \frac{|\Omega(y-x)|\left(|y-x|^{\alpha}+r^{\alpha}\right) d y}{|y-x|^{n-\sigma}}\right)^{2} \frac{d t}{t}\right)^{1 / 2} \\
& \leq C\|\Omega\|_{L^{1}\left(S^{n-1}\right)}\|f\|_{\operatorname{Lip}_{\alpha}}\left(\int_{r}^{\infty}\left(\frac{1}{t^{\sigma}} \int_{t-r}^{t} \frac{\left(s^{\alpha}+r^{\alpha}\right) s^{n-1} d s}{s^{n-\sigma}}\right)^{2} \frac{d t}{t}\right)^{1 / 2} \\
& \leq C\|\Omega\|_{L^{1}\left(S^{n-1}\right)}\|f\|_{\operatorname{Lip}_{\alpha}}\left(\int_{r}^{\infty}\left(\frac{1}{t^{\sigma}}\left(r t^{\sigma+\alpha-1}+r^{\alpha} r t^{\sigma-1}\right)\right)^{2} \frac{d t}{t}\right)^{1 / 2} \\
& \leq C\|\Omega\|_{L^{1}\left(S^{n-1}\right)}\|f\|_{\operatorname{Lip}_{\alpha}} r^{\alpha} .
\end{aligned}
$$

Similarly, we can get the same estimate for $I_{2}(x)$.

As for $I_{3}(x)$, we see easily as before that $\left|f_{3}(y)\right| \leq C\|f\|_{\operatorname{Lip}_{\alpha}} t^{\alpha}$ for $|y-x|<t,\left|y-x_{0}\right|<t$ and $t>r$. Using Lemma 1.2 we have

$$
\begin{aligned}
& J(r, t):=\int_{\substack{|y-x|<t \\
\left|y-x_{0}\right|<t}}\left|\frac{\Omega(y-x)}{|y-x|^{n-\rho}}-\frac{\Omega\left(y-x_{0}\right)}{\left|y-x_{0}\right|^{n-\rho}}\right|\left|f_{3}(y)\right| d y \\
& \leq C\|f\|_{\operatorname{Lip}_{\alpha}} t^{\alpha} \int_{4 r<\left|y-x_{0}\right|<2 t}\left|\frac{\Omega(y-x)}{|y-x|^{n-\rho}}-\frac{\Omega\left(y-x_{0}\right)}{\left|y-x_{0}\right|^{n-\rho}}\right| d y \\
& \leq C\|f\|_{\operatorname{Lip}_{\alpha}} t^{\alpha} \sum_{k=2}^{\left[\log _{2} \frac{t}{r}\right]+1} \int_{2^{k} r \leq\left|y-x_{0}\right|<2^{k+1} r}\left|\frac{\Omega(y-x)}{|y-x|^{n-\rho}}-\frac{\Omega\left(y-x_{0}\right)}{\left|y-x_{0}\right|^{n-\rho}}\right| d y \\
& \leq C\|f\|_{\operatorname{Lip}_{\alpha}} t^{\alpha} \sum_{k=2}^{\left[\log _{2} \frac{t}{r}\right]+1}\left(2^{k} r\right)^{n-(n-\sigma)}\left(\|\Omega\|_{L^{1}\left(S^{n-1}\right)} \frac{r}{2^{k} r}+\int_{r /\left(2^{k+1} r\right)}^{r /\left(2^{k} r\right)} \frac{\omega_{1}(\delta)}{\delta} d \delta\right) \\
& \leq C\|f\|_{\operatorname{Lip}_{\alpha}} t^{\alpha} \sum_{k=2}^{\left[\log _{2} \frac{t}{r}\right]+1} r^{\sigma}\left(\|\Omega\|_{L^{1}\left(S^{n-1}\right)} 2^{(\sigma-1) k}+2^{(\sigma-\beta) k} \int_{2^{-k-1}}^{2^{-k}} \frac{\omega_{1}(\delta)}{\delta^{1+\beta}} d \delta\right) .
\end{aligned}
$$


To continue estimating $J(r, t)$, we treat the following four cases.

(a) $\sigma>1$. Since $\sigma>1$, we see that

$$
\sum_{k=2}^{\left[\log _{2} \frac{t}{r}\right]+1}\left(2^{(\sigma-1) k}+2^{(\sigma-\beta) k}\right) \leq C\left(\left(\frac{t}{r}\right)^{\sigma-1}+\left(\frac{t}{r}\right)^{\sigma-\beta}\right)
$$

and hence we have

$$
J(r, t) \leq C\|f\|_{\operatorname{Lip}_{\alpha}}\left(r t^{\sigma+\alpha-1}+r^{\beta} t^{\sigma+\alpha-\beta}\right) .
$$

Thus, noting $\alpha<\beta \leq 1$, we have

$$
I_{3}(x) \leq C\|f\|_{\operatorname{Lip}_{\alpha}}\left(\int_{r}^{\infty}\left(r^{2} t^{2 \alpha-2}+r^{2 \beta} t^{2 \alpha-2 \beta}\right) \frac{d t}{t}\right)^{1 / 2} \leq C\|f\|_{\operatorname{Lip}_{\alpha}} r^{\alpha} .
$$

(b) $\sigma=1$. In this case, we have

$$
\begin{aligned}
J(r, t) & \leq C\|f\|_{\operatorname{Lip}_{\alpha}} t^{\alpha} r^{\sigma}\left(\|\Omega\|_{L^{1}\left(S^{n-1}\right)} \log _{2} \frac{t}{r}+\left(\frac{t}{r}\right)^{\sigma-\beta} \int_{0}^{1} \frac{\omega_{1}(\delta)}{\delta^{1+\beta}} d \delta\right) \\
& \leq C\|f\|_{\operatorname{Lip}_{\alpha}} t^{\alpha} r^{\sigma}\left(\log _{2} \frac{t}{r}+\left(\frac{t}{r}\right)^{\sigma-\beta}\right) .
\end{aligned}
$$

Thus, noting $\alpha<\beta \leq 1$, we have

$$
\begin{aligned}
I_{3}(x) & \leq C\|f\|_{\operatorname{Lip}_{\alpha}}\left(\int_{r}^{\infty} t^{2 \alpha}\left(\left(\frac{r}{t}\right)^{2 \sigma} \log _{2}^{2} \frac{t}{r}+\left(\frac{r}{t}\right)^{2 \beta}\right) \frac{d t}{t}\right)^{1 / 2} \\
& \leq C\|f\|_{\operatorname{Lip}_{\alpha}} r^{\alpha}\left(\int_{1}^{\infty}\left(\frac{1}{s^{2 \sigma-2 \alpha+1}} \log _{2}^{2} s+\frac{1}{s^{2 \beta-2 \alpha+1}}\right) d s\right)^{1 / 2} \\
& =C\|f\|_{\operatorname{Lip}_{\alpha}} r^{\alpha} .
\end{aligned}
$$

(c) $0<\beta<\sigma<1$. In this case, we have

$$
J(r, t) \leq C\|f\|_{\operatorname{Lip}_{\alpha}} t^{\alpha} r^{\sigma}\left(\|\Omega\|_{L^{1}\left(S^{n-1}\right)}+\left(\frac{t}{r}\right)^{\sigma-\beta} \int_{0}^{1} \frac{\omega_{1}(\delta)}{\delta^{1+\beta}} d \delta\right) .
$$

Thus, noting $\alpha<\beta<\sigma$, we have

$$
\begin{aligned}
I_{3}(x) & \leq C\|f\|_{\operatorname{Lip}_{\alpha}}\left(\int_{r}^{\infty} t^{2 \alpha}\left(\left(\frac{r}{t}\right)^{2 \sigma}+\left(\frac{r}{t}\right)^{2 \beta}\right) \frac{d t}{t}\right)^{1 / 2} \\
& \leq C\|f\|_{\operatorname{Lip}_{\alpha}} r^{\alpha}\left(\int_{1}^{\infty}\left(\frac{1}{s^{2 \sigma-2 \alpha+1}}+\frac{1}{s^{2 \beta-2 \alpha+1}}\right) d s\right)^{1 / 2} \\
& =C\|f\|_{\operatorname{Lip}_{\alpha}} r^{\alpha} .
\end{aligned}
$$


(d) $0<\sigma \leq \beta \leq 1$ and $\sigma<1$. In this case, we have

$$
J(r, t) \leq C\|f\|_{\operatorname{Lip}_{\alpha}} t^{\alpha} r^{\sigma}\left(\|\Omega\|_{L^{1}\left(S^{n-1}\right)}+\int_{0}^{1} \frac{\omega_{1}(\delta)}{\delta^{1+\beta}} d \delta\right) .
$$

Thus, noting $\alpha<\sigma$, we have

$$
\begin{aligned}
I_{3}(x) & \leq C\|f\|_{\operatorname{Lip}_{\alpha}}\left(\int_{r}^{\infty} t^{2 \alpha}\left(\frac{r}{t}\right)^{2 \sigma} \frac{d t}{t}\right)^{1 / 2} \\
& \leq C\|f\|_{\operatorname{Lip}_{\alpha}} r^{\alpha}\left(\int_{1}^{\infty} \frac{1}{s^{2 \sigma-2 \alpha+1}} d s\right)^{1 / 2}=C\|f\|_{\operatorname{Lip}_{\alpha}} r^{\alpha} .
\end{aligned}
$$

Summing up the estimates for $I_{1}(x), I_{2}(x), I_{3}(x)$, we obtain the desired estimate in the case (ii).

(iii) Take a positive constant $\eta$ with $0<\eta<p-1$. For $y \in(4 B)^{c}$, $|y-x|<t, x \in B$, we have $t>\left|y-x_{0}\right|-\left|x-x_{0}\right|>3 r$. Noting this, we get by Hölder's inequality, Lemma 1.3 and Lemma 1.1

$$
\begin{aligned}
& \int_{\substack{|y-x|<t \\
\left|y-x_{0}\right| \geq t}} \frac{\left|\Omega(y-x) f_{3}(y)\right| d y}{|y-x|^{n-\sigma}} \\
& \leq\left(\int_{\max \{3 r, t-r\}<|y-x|<t} \frac{|\Omega(y-x)|^{p^{\prime}} d y}{|y-x|^{(n-\sigma)^{p^{\prime}}-(n+\eta) p^{\prime} / p}}\right)^{1 / p^{\prime}}\left(\int_{\mathbb{R}^{n}} \frac{\left|f_{3}(y)\right|^{p} d y}{|y-x|^{n+\eta}}\right)^{1 / p} \\
& \leq\|\Omega\|_{L^{p^{\prime}}\left(S^{n-1}\right)}\left(\int_{\max \{3 r, t-r\}}^{t} s^{(n+\eta) p^{\prime} / p-(n-\sigma) p^{\prime}+n-1} d s\right)^{1 / p^{\prime}}\|f\|_{\mathcal{E}^{\alpha, p}} r^{\alpha-\eta / p} \\
& \leq C\|\Omega\|_{L^{p^{\prime}\left(S^{n-1}\right)}}\|f\|_{\mathcal{E}^{\alpha, p} r^{\alpha-\eta / p} r^{1 / p^{\prime}} t^{\eta / p-1 / p^{\prime}+\sigma}} .
\end{aligned}
$$

Thus, we have, noting $\eta / p-1 / p^{\prime}=(\eta-(p-1)) / p<0$,

$$
I_{1}(x) \leq C\|f\|_{\mathcal{E}^{\alpha, p}} r^{\alpha-\eta / p+1 / p^{\prime}}\left(\int_{3 r}^{\infty} t^{2 \eta / p-2 / p^{\prime}-1} d t\right)^{1 / 2} \leq C\|f\|_{\mathcal{E}^{\alpha, p}} r^{\alpha} .
$$

Similarly, we have the same estimate for $I_{2}(x)$.

As for $I_{3}(x)$, we have by Minkowski's inequality and Hölder's inequality

$$
\begin{aligned}
I_{3}(x) & \leq \int_{\mathbb{R}^{n}}\left|\frac{\Omega(y-x)}{|y-x|^{n-\rho}}-\frac{\Omega\left(y-x_{0}\right)}{\left|y-x_{0}\right|^{n-\rho}}\right|\left|f_{3}(y)\right|\left(\int_{r}^{\infty} \chi_{\left|y-x_{0}\right|<t} \frac{d t}{t^{2 \sigma+1}}\right)^{1 / 2} d y \\
& \leq C \int_{\left|y-x_{0}\right|>4 r}\left|\frac{\Omega(y-x)}{|y-x|^{n-\rho}}-\frac{\Omega\left(y-x_{0}\right)}{\left|y-x_{0}\right|^{n-\rho}}\right| \frac{\left|f_{3}(y)\right|}{\left|y-x_{0}\right|^{\sigma}} d y
\end{aligned}
$$




$$
\begin{gathered}
=\sum_{j=2}^{\infty} \int_{2^{j} r \leq\left|y-x_{0}\right|<2^{j+1} r}\left|\frac{\Omega(y-x)}{|y-x|^{n-\rho}}-\frac{\Omega\left(y-x_{0}\right)}{\left|y-x_{0}\right|^{n-\rho}}\right| \frac{\left|f_{3}(y)\right|}{\left|y-x_{0}\right|^{\sigma}} d y \\
\leq \sum_{j=2}^{\infty}\left(\int_{2^{j} r \leq\left|y-x_{0}\right|<2^{j+1} r}\left|\frac{\Omega(y-x)}{|y-x|^{n-\rho}}-\frac{\Omega\left(y-x_{0}\right)}{\left|y-x_{0}\right|^{n-\rho}}\right|^{p^{\prime}} d y\right)^{1 / p^{\prime}} \\
\times\left(\int_{2^{j} r \leq\left|y-x_{0}\right|<2^{j+1} r} \frac{\left|f_{3}(y)\right|^{p}}{\left|y-x_{0}\right|^{p \sigma}} d y\right)^{1 / p} .
\end{gathered}
$$

Now, since $\alpha<0$ and $f \in \mathcal{E}^{\alpha, p}$, we see easily

$$
\begin{aligned}
& \left(\frac{1}{\left|2^{j+1} B\right|} \int_{2^{j} r \leq\left|y-x_{0}\right|<2^{j+1} r} \frac{\left|f_{3}(y)\right|^{p}}{\left|y-x_{0}\right|^{p \sigma}} d y\right)^{1 / p} \\
& \quad \leq \frac{1}{\left(2^{j} r\right)^{\sigma}}\left(\frac{1}{\left|2^{j+1} B\right|} \int_{2^{j+1} B}\left|f(y)-f_{4 B}\right|^{p} d y\right)^{1 / p} \\
& \leq C\|f\|_{\mathcal{E}^{\alpha, p} r^{\alpha-\sigma} 2^{-j \sigma}} .
\end{aligned}
$$

Hence, using Lemma 1.2, we get

$$
\begin{aligned}
I_{3}(x) \leq C \sum_{j=2}^{\infty}\left(2^{j} r\right)^{n / p^{\prime}-(n-\sigma)}\left(\|\Omega\|_{L^{p^{\prime}}\left(S^{n-1}\right)} \frac{\left|x-x_{0}\right|}{2^{j} r}+\int_{\frac{\left|x-x_{0}\right|}{2^{j+1} r}}^{\frac{\left|x-x_{0}\right|}{2^{j} r}} \frac{\omega_{p^{\prime}}(\delta)}{\delta} d \delta\right) \\
\quad \times\|f\|_{\mathcal{E}^{\alpha, p}} 2^{-j \sigma} r^{\alpha-\sigma}\left(2^{j} r\right)^{n / p} \\
\leq C r^{\alpha}\|f\|_{\mathcal{E}^{\alpha, p}} \sum_{j=2}^{\infty}\left(\|\Omega\|_{L^{p^{\prime}}\left(S^{n-1}\right)} 2^{-j}+\int_{\frac{\left|x-x_{0}\right|}{2^{j+1} r}}^{\frac{\left|x-x_{0}\right|}{2^{j} r}} \frac{\omega_{p^{\prime}}(\delta)}{\delta} d \delta\right) \\
\leq C r^{\alpha}\|f\|_{\mathcal{E}^{\alpha, p}}\left(\|\Omega\|_{L^{p^{\prime}\left(S^{n-1}\right)}}+\int_{0}^{1} \frac{\omega_{p^{\prime}}(\delta)}{\delta} d \delta\right) .
\end{aligned}
$$

Summing up the estimates for $I_{1}(x), I_{2}(x), I_{3}(x)$, we obtain the desired estimate in the case (iii).

Next, we prepare three lemmas for the proofs of Theorems 4, 5, and 6 . As for $\mu_{S, \infty}^{\rho}\left(f_{2}\right)$ we have

LEMMA 2.4. Let $\rho=\sigma+i \tau$ with $\sigma>0$ and $\tau \in \mathbb{R}$.

(a) Let $\max \left\{1, \frac{2 n}{n+2 \sigma}\right\}<p<+\infty$ and $-n / p \leq \alpha<0$. Moreover, $\Omega \in L^{p_{0}^{\prime}}\left(S^{n-1}\right)$ for $p_{0}=\min \{p, 2\}$. Then, for any $f \in \mathcal{E}^{\alpha, p}$, any ball $B=B\left(x_{0}, r\right)$ and any $x \in \mathbb{R}^{n}$

$$
\mu_{S, \infty}^{\rho}\left(f_{2}\right)(x) \leq C\|\Omega\|_{L^{p_{0}^{\prime}\left(S^{n-1}\right)}} r^{\alpha}\|f\|_{\mathcal{E}^{\alpha, p}}
$$


(b) Let $1 \leq p<\infty, \alpha=0$ and $\Omega \in L^{q}\left(S^{n-1}\right)$ for some $q>1$. Then, for any $f \in \mathcal{E}^{\alpha, p}$, any ball $B=B\left(x_{0}, r\right)$ and any $x \in \mathbb{R}^{n}$

$$
\mu_{S, \infty}^{\rho}\left(f_{2}\right)(x) \leq C\|\Omega\|_{L^{q}\left(S^{n-1}\right)}\|f\|_{\mathcal{E}^{\alpha, p}}
$$

(c) Let $1 \leq p<\infty, 0<\alpha<1$, and $\Omega \in L^{1}\left(S^{n-1}\right)$. Then, for any $f \in \mathcal{E}^{\alpha, p}$, any ball $B=B\left(x_{0}, r\right)$ and any $x \in \mathbb{R}^{n}$

$$
\mu_{S, \infty}^{\rho}\left(f_{2}\right)(x) \leq C\|\Omega\|_{L^{1}\left(S^{n-1}\right)}\|f\|_{\mathcal{E}^{\alpha, p}} r^{\alpha}
$$

Since one can prove this lemma more easily than the corresponding lemma for $\mu_{\lambda, \infty}^{*, \rho}\left(f_{2}\right)(x)$, we omit the proof of this lemma.

Lemma 2.5. Let $\Omega \in L^{1}\left(S^{n-1}\right), \rho \in \mathbb{C}$. Then, for any $f \in L_{\text {loc }}^{1}\left(\mathbb{R}^{n}\right)$, for any ball $B=B\left(x_{0}, r\right)$ and any $x \in B$

$$
\mu_{S, 0}^{\rho}\left(f_{3}\right)(x)=\left(\int_{0}^{r} \int_{|u-x| \leq t}\left|\frac{1}{t^{\rho}} \int_{|y-u| \leq t} \frac{\Omega(u-y) f_{3}(y)}{|u-y|^{n-\rho}} d y\right|^{2} \frac{d u d t}{t^{n+1}}\right)^{1 / 2}=0 .
$$

Proof. For $\left|x-x_{0}\right| \leq r,|u-x| \leq t \leq r$ and $|u-y| \leq t \leq r$, we have $\left|x_{0}-y\right| \leq\left|x_{0}-x\right|+|x-u|+|u-y| \leq 3 r$, and hence the integration domain with respect to $y$ has no intersection with the support of $f_{3}$ in the expression of $\mu_{S, 0}^{\rho}\left(f_{3}\right)$. So, we have $\mu_{S, 0}^{\rho}\left(f_{3}\right)=0$ for $x \in B$.

LEMMA 2.6. Suppose one of the following five conditions is satisfied:

(i) $\sigma>0, \alpha=0,1 \leq p<\infty$. Moreover, $\Omega \in L^{q}\left(S^{n-1}\right)$ for some $q>1$ and satisfies the cancellation condition.

(ii) $\sigma>0,0<\beta \leq 1,0<\alpha<\min \{1 / 2, \min \{\beta, \sigma\}\}$, and $1 \leq p<\infty$. Moreover, $\Omega \in L^{1}\left(S^{n-1}\right)$ and satisfies the cancellation condition.

(iii) $\sigma>0,0<\beta \leq 1,1 / 2 \leq \alpha<\min \{\beta, \sigma\}$, and $1 \leq p<\infty$. Moreover, $\Omega \in L^{1}\left(S^{n-1}\right)$ and satisfies $L^{1}-\beta$-Dini condition and the cancellation condition.

(iv) $\max \left\{1, \frac{2 n}{n+2 \sigma}\right\}<p<+\infty,-n / p<\alpha<0$, and $\sigma>-\alpha$. Moreover, $\Omega \in L^{p_{0}^{\prime}}\left(S^{n-1}\right)$ and satisfies the cancellation condition, where $p_{0}=$ $\max \{2, p\}$.

(v) $1<p<\infty,-n / p \leq \alpha<0$, and $\sigma>n / 2$. Moreover, $\Omega \in L^{2}\left(S^{n-1}\right)$ and satisfies $L^{2}-\log \beta$ Dini condition for some $\beta>1$ and the cancellation condition. 
Then there exists $C>0$ such that for any ball $B=B\left(x_{0}, r\right)$ and any $f \in \mathcal{E}^{\alpha, p}$ satisfying $\mu_{S, \infty}^{\rho}\left(f_{3}\right)\left(x_{0}\right)<+\infty$, it holds

$$
\mu_{S, \infty}^{\rho}\left(f_{3}\right)(x)<+\infty \quad \text { and } \quad\left|\mu_{S, \infty}^{\rho}\left(f_{3}\right)(x)-\mu_{S, \infty}^{\rho}\left(f_{3}\right)\left(x_{0}\right)\right| \leq C r^{\alpha}\|f\|_{\mathcal{E}^{\alpha, p}}
$$

for any $x \in B$, where $f_{3}(x)=\left(f(x)-f_{4 B}\right) \chi_{(4 B)^{c}}$.

Since one can prove this lemma more easily than the corresponding lemma for $\mu_{\lambda, \infty}^{*, \rho}\left(f_{3}\right)(x)$, we omit the proof of this lemma.

Finally, we prepare six lemmas for the proofs of Theorems 7, 8, and 9.

LEMMA 2.7. Let $\rho=\sigma+i \tau(\sigma>0, \tau \in \mathbb{R})$, and $\lambda>1$.

(a) Let $\max \left\{1, \frac{2 n}{n+2 \sigma}\right\}<p<+\infty$ and $-n / p \leq \alpha<0$. Moreover, $\Omega \in L^{p_{0}^{\prime}}\left(S^{n-1}\right)$ for $p_{0}=\min \{p, 2\}$. Then, for any $f \in \mathcal{E}^{\alpha, p}$, any ball $B=B\left(x_{0}, r\right)$ and any $x \in \mathbb{R}^{n}$

$$
\mu_{\lambda, \infty}^{*, \rho}\left(f_{2}\right)(x) \leq C\|\Omega\|_{L^{p_{0}^{\prime}\left(S^{n-1}\right)}} r^{\alpha}\|f\|_{\mathcal{E}^{\alpha, p}}
$$

(b) Let $1 \leq p<\infty, \alpha=0$ and $\Omega \in L^{q}\left(S^{n-1}\right)$ for some $q>1$. Then, for any $f \in \mathcal{E}^{\alpha, p}$, any ball $B=B\left(x_{0}, r\right)$ and any $x \in \mathbb{R}^{n}$

$$
\mu_{\lambda, \infty}^{*, \rho}\left(f_{2}\right)(x) \leq C\|\Omega\|_{L^{q}\left(S^{n-1}\right)}\|f\|_{\mathcal{E}^{\alpha, p}}
$$

(c) Let $1 \leq p<\infty, 0<\alpha<1$, and $\Omega \in L^{1}\left(S^{n-1}\right)$. Then, for any $f \in \mathcal{E}^{\alpha, p}$, any ball $B=B\left(x_{0}, r\right)$ and any $x \in \mathbb{R}^{n}$

$$
\mu_{\lambda, \infty}^{*, \rho}\left(f_{2}\right)(x) \leq C\|\Omega\|_{L^{1}\left(S^{n-1}\right)}\|f\|_{\mathcal{E}^{\alpha, p}} r^{\alpha}
$$

Proof. Proof of (a).

(i) The case $0<\sigma<n$ and $\max \left\{1, \frac{2 n}{n+2 \sigma}\right\}<p<+\infty$. Since the support of $f_{2}$ is contained in $4 B=\left\{\left|y-x_{0}\right|<4 r\right\}$ we see easily

$$
\begin{aligned}
& \left|\int_{r<|y-u| \leq t} \frac{\Omega(u-y) f_{2}(y)}{|u-y|^{n-\rho}} d y\right| \\
& \leq\left(\int_{r<|y-u| \leq t} \frac{|\Omega(u-y)|^{p^{\prime}} \chi_{4 B}}{|u-y|^{p^{\prime}(n-\sigma)}} d y\right)^{1 / p^{\prime}}\left(\int_{\left|y-x_{0}\right| \leq 4 r}\left|f(y)-f_{4 B}\right|^{p} d y\right)^{1 / p} \\
& \leq C\|\Omega\|_{L^{p^{\prime}\left(S^{n-1}\right)}}\left(\int_{\max \left\{r,\left|u-x_{0}\right|-4 r\right\}}^{\min \left\{t,\left|u-x_{0}\right|+4 r\right\}} s^{-(n-\sigma) p^{\prime}+n-1} d s\right)^{1 / p^{\prime}} r^{\alpha+n / p}\|f\|_{\mathcal{E}^{\alpha, p}} \\
& \leq C\|\Omega\|_{L^{p^{\prime}\left(S^{n-1}\right)}}\left(r \max \left\{t^{n-(n-\sigma) p^{\prime}-1}, r^{n-(n-\sigma) p^{\prime}-1}\right\}\right)^{1 / p^{\prime}} r^{\alpha+n / p}\|f\|_{\mathcal{E}^{\alpha, p} .}
\end{aligned}
$$


In the last inequality, we use the fact: $L^{p_{0}^{\prime}}\left(S^{n-1}\right) \subset L^{p^{\prime}}\left(S^{n-1}\right)$ by $p_{0}^{\prime} \geq p^{\prime}$ and $\|\Omega\|_{L^{p^{\prime}\left(S^{n-1}\right)}} \leq C\|\Omega\|_{L^{p^{\prime}\left(S^{n-1}\right)}}$. In the case $n-(n-\sigma) p^{\prime}-1 \geq 0$, we have $\max \left\{t^{n-(n-\sigma) p^{\prime}-1}, r^{n-(n-\sigma) p^{\prime}-1}\right\}=t^{n-(n-\sigma) p^{\prime}-1}$, and hence

$$
\begin{aligned}
& \left(\int_{r}^{\infty} \int_{\mathbb{R}^{n}}\left|\frac{1}{t^{\rho}} \int_{r<|y-u| \leq t} \frac{\Omega(u-y) f_{2}(y)}{|u-y|^{n-\rho}} d y\right|^{2}\left(1+\frac{|u-x|}{t}\right)^{-\lambda n} \frac{d u d t}{t^{n+1}}\right)^{1 / 2} \\
& \leq C\|\Omega\|_{L^{p^{\prime}\left(S^{n-1}\right)}}\|f\|_{\mathcal{E}^{\alpha, p}} r^{\alpha+\frac{n}{p}} r^{\frac{1}{p^{\prime}}} \\
& \times\left(\int_{r}^{\infty} \int_{\mathbb{R}^{n}}\left(1+\frac{|u-x|}{t}\right)^{-\lambda n} \frac{d u}{t^{n}} \frac{d t}{t^{2 n\left(1-\frac{1}{p^{\prime}}\right)+\frac{2}{p^{\prime}}+1}}\right)^{1 / 2}
\end{aligned}
$$

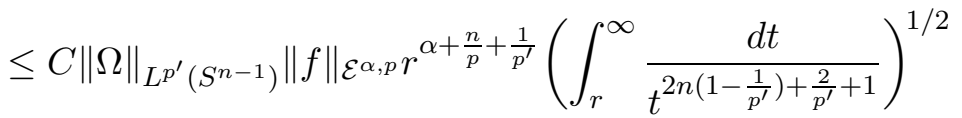

$$
\begin{aligned}
& \leq C r^{\alpha}\|\Omega\|_{L^{p^{\prime}\left(S^{n-1}\right)}}\|f\|_{\mathcal{E}^{\alpha, p} .}
\end{aligned}
$$

And in the case $n-(n-\sigma) p^{\prime}-1<0$, we have $\max \left\{t^{n-(n-\sigma) p^{\prime}-1}\right.$, $\left.r^{n-(n-\sigma) p^{\prime}-1}\right\}=r^{n-(n-\sigma) p^{\prime}-1}$, and hence

$$
\begin{aligned}
& \left(\int_{r}^{\infty} \int_{\mathbb{R}^{n}}\left|\frac{1}{t^{\rho}} \int_{r<|y-u| \leq t} \frac{\Omega(u-y) f_{2}(y)}{|u-y|^{n-\rho}} d y\right|^{2}\left(1+\frac{|u-x|}{t}\right)^{-\lambda n} \frac{d u d t}{t^{n+1}}\right)^{1 / 2} \\
& \leq C\|\Omega\|_{L^{p^{\prime}}\left(S^{n-1}\right)}\|f\|_{\mathcal{E}^{\alpha, p} r^{\alpha+\frac{n}{p}} r^{\frac{1}{p^{\prime}}} r^{\frac{n}{p^{\prime}}-(n-\sigma)-\frac{1}{p^{\prime}}}} \\
& \quad \times\left(\int_{r}^{\infty} \int_{\mathbb{R}^{n}}\left(1+\frac{|u-x|}{t}\right)^{-\lambda n} \frac{d u}{t^{n}} \frac{d t}{t^{2 \sigma+1}}\right)^{1 / 2} \\
& \leq C\|\Omega\|_{L^{p^{\prime}\left(S^{n-1}\right)}}\|f\|_{\mathcal{E}^{\alpha, p} r^{\alpha+\sigma}\left(\int_{r}^{\infty} \frac{d t}{t^{2 \sigma+1}}\right)^{1 / 2}} \leq C r^{\alpha}\|\Omega\|_{L^{p_{0}^{\prime}\left(S^{n-1}\right)}}\|f\|_{\mathcal{E}^{\alpha, p} .}
\end{aligned}
$$

So, we need only to show

$$
\begin{aligned}
I & :=\left(\int_{r}^{\infty} \int_{\mathbb{R}^{n}}\left|\frac{1}{t^{\rho}} \int_{|y-u| \leq r} \frac{\Omega(u-y) f_{2}(y)}{|u-y|^{n-\rho}} d y\right|^{2}\left(1+\frac{|u-x|}{t}\right)^{-\lambda n} \frac{d u d t}{t^{n+1}}\right)^{1 / 2} \\
& \leq C r^{\alpha}\|\Omega\|_{L^{p^{\prime}\left(S^{n-1}\right)}}\|f\|_{\mathcal{E}^{\alpha, p} .}
\end{aligned}
$$

Since $p>\frac{2 n}{n+2 \sigma}$, we have

$$
\frac{n}{2(n-\sigma)}-\left(1-\frac{n}{n-\sigma}\left(1-\frac{1}{p}\right)\right)=\frac{n+2 \sigma}{2 p(n-\sigma)}\left(p-\frac{2 n}{n+2 \sigma}\right)>0 \text {. }
$$


So, we take $p_{0}=\min \{2, p\}$ and choose a real number $a$ so that

$$
\min \left\{1, \frac{n}{2(n-\sigma)}\right\}>a>1-\frac{n}{(n-\sigma) p_{0}^{\prime}}, \quad \text { where } \quad \frac{1}{p_{0}}+\frac{1}{p_{0}^{\prime}}=1
$$

Then, noting $0<(n-\sigma)(1-a) p_{0}^{\prime}<n$ we have by Hölder's inequality

$$
\begin{aligned}
& \left|\int_{|y-u| \leq r} \frac{\Omega(u-y) f_{2}(y)}{|u-y|^{n-\rho}} d y\right| \\
& \quad \leq\left(\int_{|y-u| \leq r} \frac{|\Omega(u-y)|^{p_{0}^{\prime}} d y}{|u-y|^{(n-\sigma)(1-a) p_{0}^{\prime}}}\right)^{1 / p_{0}^{\prime}}\left(\int_{|y-u| \leq r} \frac{\left|f_{2}(y)\right|^{p_{0}} d y}{|u-y|^{(n-\sigma) a p_{0}}}\right)^{1 / p_{0}} \\
& \quad \leq C\|\Omega\|_{L^{p^{\prime}\left(S^{n-1}\right)}} r^{\frac{n}{p_{0}^{\prime}}-(n-\sigma)(1-a)}\left(\int_{|y-u| \leq r} \frac{\left|f_{2}(y)\right|^{p_{0}} d y}{|u-y|^{(n-\sigma) a p_{0}}}\right)^{1 / p_{0}} .
\end{aligned}
$$

Hence by Minkowski's inequality $\left(2 / p_{0} \geq 1\right)$ and by using $2 a(n-\sigma)<n$ we get

$$
\begin{aligned}
& I /\|\Omega\|_{L^{p_{0}^{\prime}\left(S^{n-1}\right)}} \leq C r^{\frac{n}{p_{0}^{\prime}}-(n-\sigma)(1-a)} \\
& \quad \times\left(\int_{r}^{\infty}\left(\int_{4 B}\left(\int_{|y-u| \leq r} \frac{\left(\frac{t}{t+|u-x|}\right)^{\lambda n} d u}{|u-y|^{2(n-\sigma) a}}\right)^{p_{0} / 2}\left|f_{2}(y)\right|^{p_{0}} d y\right)^{2 / p_{0}} \frac{d t}{t^{2 \sigma+n+1}}\right)^{1 / 2} \\
& \leq C r^{\frac{n}{p_{0}^{\prime}}-(n-\sigma)(1-a)} r^{\frac{n}{2}-(n-\sigma) a}\left(\int_{r}^{\infty}\left(\int_{4 B}\left|f(y)-f_{4 B}\right|^{p_{0}} d y\right)^{2 / p_{0}} \frac{d t}{t^{2 \sigma+n+1}}\right)^{1 / 2} \\
& \leq C r^{\frac{n}{p_{0}^{\prime}}+\frac{n}{2}-(n-\sigma)} r^{\alpha+\frac{n}{p_{0}}}\|f\|_{\mathcal{E}^{\alpha, p_{0}} r^{-\sigma-\frac{n}{2}} \leq C r^{\alpha}\|f\|_{\mathcal{E}^{\alpha, p} .}}
\end{aligned}
$$

(ii) The case $\sigma \geq n$. In this case we see easily

$$
\begin{aligned}
& \left|\int_{|y-u| \leq t} \frac{\Omega(u-y) f_{2}(y)}{|u-y|^{n-\rho}} d y\right| \leq t^{\sigma-n} \int_{|y-u| \leq t}|\Omega(u-y)|\left|f_{2}(y)\right| d y \\
& \leq t^{\sigma-n}\left(\int_{|y-u| \leq t}|\Omega(u-y)|^{p^{\prime}} d y\right)^{1 / p^{\prime}}\left(\int_{\left|y-x_{0}\right| \leq 4 r}\left|f(y)-f_{4 B}\right|^{p} d y\right)^{1 / p} \\
& \leq C t^{\sigma-n+n / p^{\prime}}\|\Omega\|_{L^{p^{\prime}\left(S^{n-1}\right)}} r^{\alpha+n / p}\|f\|_{\mathcal{E}^{\alpha, p} .}
\end{aligned}
$$


Using this, we get

$$
\begin{aligned}
& \mu_{\lambda, \infty}^{*, \rho}\left(f_{2}\right)(x) /\left(\|\Omega\|_{L^{p_{0}^{\prime}\left(S^{n-1}\right)}}\|f\|_{\left.\mathcal{E}^{\alpha, p}\right)}\right. \\
& \leq C r^{\alpha+n / p}\left(\int_{r}^{\infty} \int_{\mathbb{R}^{n}} t^{2\left(-n+\frac{n}{p^{\prime}}\right)}\left(1+\frac{|u-x|}{t}\right)^{-\lambda n} \frac{d u d t}{t^{n+1}}\right)^{1 / 2} \\
& \leq C r^{\alpha+n / p}\left(\int_{r}^{\infty}\left(\int_{\mathbb{R}^{n}}\left(1+\frac{|u-x|}{t}\right)^{-\lambda n} \frac{d u}{t^{n}}\right) \frac{d t}{t^{2 n\left(1-\frac{1}{p^{\prime}}\right)+1}}\right)^{1 / 2} \\
& \leq C r^{\alpha+n / p} r^{-n\left(1-\frac{1}{p^{\prime}}\right)} \leq C r^{\alpha} .
\end{aligned}
$$

Proof of (b). In this case, $\mathcal{E}^{\alpha, p}=\mathrm{BMO}(1 \leq p<\infty)$, and the norms are equivalent. Choose a positive number $q_{0}$ such that $q_{0} \leq q$ and $(n-\sigma) q_{0}<n$. Then, using the Hölder inequality, the increasingness of $s^{n-(n-\sigma) q_{0}}$ and the decreasingness of $s^{-1}$, we have

$$
\begin{aligned}
& \left|\int_{r<|y-u| \leq t} \frac{\Omega(u-y) f_{2}(y)}{|u-y|^{n-\rho}} d y\right| \\
& \quad \leq\left(\int_{r<|y-u| \leq t} \frac{|\Omega(u-y)|^{q_{0}} \chi_{4 B}}{|u-y|^{(n-\sigma) q_{0}}} d y\right)^{1 / q_{0}}\left(\int\left|f_{2}(y)\right|^{q_{0}^{\prime}} d y\right)^{1 / q_{0}^{\prime}} \\
& \quad \leq C\|\Omega\|_{L^{q_{0}\left(S^{n-1}\right)}}\left(\int_{\max \left\{r,\left|u-x_{0}\right|-4 r\right\}}^{\min \left\{t,\left|u-x_{0}\right|+4 r\right\}} s^{-(n-\sigma) q_{0}+n-1} d s\right)^{1 / q_{0}}\|f\|_{\mathrm{BMO}} r^{n / q_{0}^{\prime}} \\
& \quad \leq C\|\Omega\|_{L^{q_{0}\left(S^{n-1}\right)}}\|f\|_{\mathrm{BMO}} r^{n / q_{0}^{\prime}} t^{n / q_{0}-(n-\sigma)}\left(\int_{\max \left\{r,\left|u-x_{0}\right|-4 r\right\}}^{\min \left\{t,\left|u-x_{0}\right|+4 r\right\}} s^{-1} d s\right)^{1 / q_{0}} \\
& \quad \leq C\|\Omega\|_{L^{q_{0}\left(S^{n-1}\right)}}\|f\|_{\mathrm{BMO}} r^{n / q_{0}^{\prime}} t^{n / q_{0}-(n-\sigma)}\left(8 r \cdot r^{-1}\right)^{1 / q_{0}} \\
& \leq C\|\Omega\|_{L^{q}\left(S^{n-1}\right)}\|f\|_{\mathrm{BMO}} r^{n / q_{0}^{\prime}} t^{\sigma-n / q_{0}^{\prime}} .
\end{aligned}
$$

Hence

$$
\begin{gathered}
\left(\int_{r}^{\infty} \int_{\mathbb{R}^{n}}\left|\frac{1}{t^{\rho}} \int_{r<|y-u| \leq t} \frac{\Omega(u-y) f_{2}(y)}{|u-y|^{n-\rho}} d y\right|^{2}\left(1+\frac{|u-x|}{t}\right)^{-\lambda n} \frac{d u d t}{t^{n+1}}\right)^{1 / 2} \\
\leq C\|\Omega\|_{L^{q}\left(S^{n-1}\right)}\|f\|_{\mathrm{BMO}} r^{n / q_{0}^{\prime}} \\
\quad \times\left(\int_{r}^{\infty}\left(\int_{\mathbb{R}^{n}}\left(1+\frac{|u-x|}{t}\right)^{-\lambda n} \frac{d u}{t^{n}}\right) t^{-2 n / q_{0}^{\prime}} \frac{d t}{t}\right)^{1 / 2} \\
\leq C\|\Omega\|_{L^{q}\left(S^{n-1}\right)}\|f\|_{\mathrm{BMO}} r^{n / q_{0}^{\prime}} r^{-n / q_{0}^{\prime}} \leq C\|\Omega\|_{L^{q}\left(S^{n-1}\right)}\|f\|_{\mathrm{BMO}} .
\end{gathered}
$$


Further,

$$
\begin{aligned}
& \left|\int_{|y-u| \leq r} \frac{\Omega(u-y) f_{2}(y)}{|u-y|^{n-\rho}} d y\right| \\
& \leq\left(\int_{|y-u| \leq r} \frac{|\Omega(u-y)|^{q_{0}} \chi_{4 B}}{|u-y|^{(n-\sigma) q_{0}}} d y\right)^{1 / q_{0}}\left(\int\left|f_{2}(y)\right|^{q_{0}^{\prime}} d y\right)^{1 / q_{0}^{\prime}} \\
& \leq C\|\Omega\|_{L^{q_{0}\left(S^{n-1}\right)}} r^{\sigma-n+n / q_{0}}\|f\|_{\mathrm{BMO}} r^{n / q_{0}^{\prime}} \\
& \leq C\|\Omega\|_{L^{q}\left(S^{n-1}\right)}\|f\|_{\mathrm{BMO}} r^{\sigma} \text {. }
\end{aligned}
$$

Hence,

$$
\begin{aligned}
& \left(\left.\int_{r}^{\infty} \int_{\mathbb{R}^{n}} \frac{1}{t^{\rho}} \int_{|y-u| \leq r} \frac{\Omega(u-y) f_{2}(y)}{|u-y|^{n-\rho}} d y\right|^{2}\left(1+\frac{|u-x|}{t}\right)^{-\lambda n} \frac{d u d t}{t^{n+1}}\right)^{1 / 2} \\
& \leq C\|\Omega\|_{L^{q}\left(S^{n-1}\right)}\|f\|_{\mathrm{BMO}} r^{\sigma}\left(\int_{r}^{\infty}\left(\int_{\mathbb{R}^{n}}\left(1+\frac{|u-x|}{t}\right)^{-\lambda n} \frac{d u}{t^{n}}\right) t^{-2 \sigma} \frac{d t}{t}\right)^{1 / 2} \\
& \leq C\|\Omega\|_{L^{q}\left(S^{n-1}\right)}\|f\|_{\mathrm{BMO}} .
\end{aligned}
$$

This completes the proof of (b).

Proof of (c). In the case $0<\alpha<1$, we have $\mathcal{E}^{\alpha, p}=\operatorname{Lip}_{\alpha}(1 \leq p<\infty)$, and the norms are equivalent. And we have as before $\left|f_{2}(y)\right| \leq C\|f\|_{\operatorname{Lip}_{\alpha}} r^{\alpha}$ for $y \in 4 B$. Choose a positive number $\gamma$ such that $\gamma<\min \{1, \sigma\}$. Then, as in the proof of (b), we have

$$
\begin{aligned}
& \left|\int_{r<|y-u| \leq t} \frac{\Omega(u-y) f_{2}(y)}{|u-y|^{n-\rho}} d y\right| \\
& \quad \leq C\|f\|_{\operatorname{Lip}_{\alpha}} r^{\alpha} \int_{r<|y-u| \leq t} \frac{|\Omega(u-y)| \chi_{4 B}}{|u-y|^{n-\sigma}} d y \\
& \quad \leq C\|f\|_{\operatorname{Lip}_{\alpha}} r^{\alpha}\|\Omega\|_{L^{1}\left(S^{n-1}\right)} \int_{\max \left\{r,\left|u-x_{0}\right|-4 r\right\}}^{\min \left\{t,\left|u-x_{0}\right|+4 r\right\}} s^{-(n-\sigma)+n-1} d s \\
& \quad \leq C\|f\|_{\operatorname{Lip}_{\alpha}} r^{\alpha}\|\Omega\|_{L^{1}\left(S^{n-1}\right)} t^{\sigma-\gamma} \int_{\max \left\{r,\left|u-x_{0}\right|-4 r\right\}}^{\min \left\{t,\left|u-x_{0}\right|+4 r\right\}} s^{\gamma-1} d s \\
& \quad \leq C\|\Omega\|_{L^{1}\left(S^{n-1}\right)}\|f\|_{\operatorname{Lip}_{\alpha}} r^{\alpha+\gamma} t^{\sigma-\gamma} .
\end{aligned}
$$

The rest of the proof proceeds in the same line as in the proof of (b). We omit the details.

As for $\mu_{\lambda}^{*, \rho}\left(f_{2}\right)$, we need 
LEMMA 2.8. Let $1 \leq p<\infty$. Let $\Omega \in L^{1}\left(S^{n-1}\right), \rho=\sigma+i \tau(\sigma>$ $0, \tau \in \mathbb{R}), \lambda>1$ and $-n / p \leq \alpha<1$. Then, for any $f \in L_{\text {loc }}^{1}\left(\mathbb{R}^{n}\right)$, any ball $B=B\left(x_{0}, r\right)$ and any $x \in B$

$$
\mu_{\lambda, 0, \infty}^{*, \rho}\left(f_{2}\right)(x)=0 .
$$

Proof. For $\left|y-x_{0}\right| \leq 4 r,|y-u| \leq t \leq r$ and $\left|x-x_{0}\right| \leq r$, we have $|u-x| \leq|u-y|+\left|y-x_{0}\right|+\left|x_{0}-x\right| \leq 6 r$, and hence the integration $u$-domain of the above integral is empty.

Next we investigate $\mu_{\lambda}^{*, \rho}\left(f_{3}\right)$.

LEMmA 2.9. Let $\rho=\sigma+i \tau(\sigma>0, \tau \in \mathbb{R})$.

(a) Let $\max \left\{1, \frac{2 n}{n+2 \sigma}\right\}<p<\infty, p_{0}=\min \{2, p\}$, and $\Omega \in L^{p_{0}{ }^{\prime}}\left(S^{n-1}\right)$. Suppose $\lambda>\max \{1,2 / p\}$ and $-n / p \leq \alpha<0$. Then, for any $f \in \mathcal{E}^{\alpha, p}$, for any ball $B=B\left(x_{0}, r\right)$ and any $x \in B$

$$
\begin{aligned}
& \mu_{\lambda, 0}^{*, \rho}\left(f_{3}\right)(x) \\
& \quad=\left(\int_{0}^{r} \int_{\mathbb{R}^{n}}\left|\frac{1}{t^{\rho}} \int_{|y-u| \leq t} \frac{\Omega(u-y) f_{3}(y)}{|u-y|^{n-\rho}} d y\right|^{2}\left(\frac{t}{t+|u-x|}\right)^{\lambda n} \frac{d u d t}{t^{n+1}}\right)^{1 / 2} \\
& \leq C r^{\alpha}\|\Omega\|_{L^{p^{\prime}\left(S^{n-1}\right)}}\|f\|_{\mathcal{E}^{\alpha, p} .}
\end{aligned}
$$

(b) Let $1 \leq p<\infty, \alpha=0, \lambda>1$, and $\Omega \in L^{q}\left(S^{n-1}\right)$ for some $q>1$. Then, for any $f \in \mathcal{E}^{\alpha, p}$, for any ball $B=B\left(x_{0}, r\right)$ and any $x \in B$

$$
\mu_{\lambda, 0}^{*, \rho}\left(f_{3}\right)(x) \leq C r^{\alpha}\|\Omega\|_{L^{q}\left(S^{n-1}\right)}\|f\|_{\mathcal{E}^{\alpha, p}}
$$

(c) Let $1 \leq p<\infty, 0<\alpha<1, \lambda>1+2 \alpha / n$, and $\Omega \in L^{1}\left(S^{n-1}\right)$. Then, for any $f \in \mathcal{E}^{\alpha, p}$, for any ball $B=B\left(x_{0}, r\right)$ and any $x \in B$

$$
\mu_{\lambda, 0}^{*, \rho}\left(f_{3}\right)(x) \leq C r^{\alpha}\|\Omega\|_{L^{1}\left(S^{n-1}\right)}\|f\|_{\mathcal{E}^{\alpha, p}}
$$

Proof. Proof of (a).

(i) The case $0<\sigma<n$ and $\max \left\{1, \frac{2 n}{n+2 \sigma}\right\}<p<+\infty$. Take $p_{0}$ and $a$ as in the proof of Lemma 2.7. Then, by Hölder's inequality we have

$$
\begin{aligned}
& \left|\int_{|y-u| \leq t} \frac{\Omega(u-y) f_{3}(y)}{|u-y|^{n-\rho}} d y\right| \\
& \quad \leq\left(\int_{|y-u| \leq t} \frac{|\Omega(u-y)|^{p_{0}^{\prime}} d y}{|u-y|^{(n-\sigma)(1-a) p_{0}^{\prime}}}\right)^{1 / p_{0}^{\prime}}\left(\int_{|y-u| \leq t} \frac{\left|f_{3}(y)\right|^{p_{0}} d y}{|u-y|^{(n-\sigma) a p_{0}}}\right)^{1 / p_{0}} \\
& \quad \leq C\|\Omega\|_{L^{p_{0}^{\prime}\left(S^{n-1}\right)}} t^{n / p_{0}^{\prime}-(n-\sigma)(1-a)}\left(\int_{|y-u| \leq t} \frac{\left|f_{3}(y)\right|^{p_{0}} d y}{|u-y|^{(n-\sigma) a p_{0}}}\right)^{f / 1 p_{0}} .
\end{aligned}
$$


Hence using Minkowski's inequality $\left(2 / p_{0} \geq 1\right)$ and then noting $|u-x| \geq$ $\left|y-x_{0}\right|-|y-u|-\left|x_{0}-x\right|>\frac{1}{4}\left(\left|y-x_{0}\right|+r\right)$ for $|u-y| \leq t \leq r,\left|y-x_{0}\right|>4 r$ and $\left|x_{0}-x\right| \leq r$, we have

$$
\begin{aligned}
& \mu_{\lambda, 0}^{*, \rho}\left(f_{3}\right)(x) /\|\Omega\|_{L^{p_{0}^{\prime}\left(S^{n-1}\right)}} \\
& \leq C\left(\int_{0}^{r}\left(\int_{\mathbb{R}^{n}}\left(\int_{|y-u| \leq t} \frac{\left|f_{3}(y)\right|^{p_{0}} d y}{|u-y|^{(n-\sigma) a p_{0}}}\right)^{2 / p_{0}} \frac{d u}{\left(\frac{t+|u-x|}{t}\right)^{\lambda n}}\right)\right. \\
& \left.\times t^{\frac{2 n}{p_{0}^{\prime}}-2(n-\sigma)(1-a)-2 \sigma-n-1} d t\right)^{1 / 2} \\
& \leq C\left(\int_{0}^{r}\left(\int_{(4 B)^{c}}\left(\int_{\mathbb{R}^{n}} \frac{\chi_{|y-u| \leq t}}{|u-y|^{2(n-\sigma) a}} \frac{d u}{\left(\frac{t+|u-x|}{t}\right)^{\lambda n}}\right)^{p_{0} / 2}\left|f_{3}(y)\right|^{p_{0}} d y\right)^{2 / p_{0}}\right. \\
& \left.\times t^{\frac{2 n}{p_{0}^{\prime}}-2(n-\sigma)(1-a)-2 \sigma-n-1} d t\right)^{1 / 2} \\
& \leq C\left(\int_{0}^{r}\left(\int_{(4 B)^{c}}\left(\int_{\mathbb{R}^{n}} \frac{\chi_{|y-u| \leq t} d u}{|u-y|^{2(n-\sigma) a}}\right)^{p_{0} / 2} \frac{\left|f_{3}(y)\right|^{p_{0}} d y}{\left(r+\left|y-x_{0}\right|\right)^{p_{0} \lambda n / 2}}\right)^{2 / p_{0}}\right. \\
& \left.\times t^{\lambda n+\frac{2 n}{p_{0}^{\prime}}-2(n-\sigma)(1-a)-2 \sigma-n-1} d t\right)^{1 / 2} \\
& \leq C\left(\int_{0}^{r}\left(\int_{(4 B)^{c}} \frac{\left|f(y)-f_{4 B}\right|^{p_{0}} d y}{\left(r+\left|y-x_{0}\right|\right)^{p_{0} \lambda n / 2}}\right)^{2 / p_{0}} t^{n-2(n-\sigma) a}\right. \\
& \left.\times t^{\lambda n+\frac{2 n}{p_{0}^{\prime}}-2(n-\sigma)(1-a)-2 \sigma-n-1} d t\right)^{1 / 2} \\
& \leq C\left(\int_{0}^{r} t^{\lambda n+\frac{2 n}{p_{0}^{\prime}}-2(n-\sigma)-2 \sigma-1} d t\right)^{1 / 2} r^{\alpha-\left(\frac{p_{0} \lambda}{2}-1\right) \frac{n}{p_{0}}}\|f\|_{\mathcal{E}^{\alpha, p_{0}}} \\
& \leq C r^{\frac{1}{2}\left(\lambda n+\frac{2 n}{p_{0}^{\prime}}-2 n\right)} r^{\alpha-\frac{\lambda n}{2}+\frac{n}{p_{0}}}\|f\|_{\mathcal{E}^{\alpha, p_{0}}} \leq C r^{\alpha}\|f\|_{\mathcal{E}^{\alpha, p}} .
\end{aligned}
$$

We have used here $\lambda n-\frac{2 n}{p_{0}}>0, \alpha<\left(\frac{\lambda}{2}-\frac{1}{p_{0}}\right) n$ and Lemma 1.1.

(ii) The case $\sigma \geq n$. In this case, we take $p_{0}=\min \{2, p\}$ and $a=0$. Then the reasoning in the step (i) still works.

Proof of (b). In this case, $\mathcal{E}^{\alpha, p}=$ BMO $(1 \leq p<\infty)$, and the norms are equivalent.

If $|u-x| \leq 2 r,\left|x-x_{0}\right| \leq r$ and $|u-y| \leq t<r$, we have $\left|y-x_{0}\right| \leq$ 


$$
\begin{aligned}
&\left|x_{0}-x\right|+|x-u|+|u-y|<4 r, \text { and hence } \\
& \mu_{\lambda, 0}^{*, \rho}\left(f_{3}\right)(x)=\left(\int_{0}^{r} \int_{|u-x|>2 r}\left|\frac{1}{t^{\rho}} \int_{|y-u| \leq t} \frac{\Omega(u-y) f_{3}(y)}{|u-y|^{n-\rho}} d y\right|^{2}\right. \\
&\left.\quad \times\left(\frac{t}{t+|u-x|}\right)^{\lambda n} \frac{d u d t}{t^{n+1}}\right)^{1 / 2} .
\end{aligned}
$$

Now choose the smallest integer $j_{0}$ satisfying $2^{j_{0}} t \geq\left|u-x_{0}\right|+4 r$ so that $B\left(u, 2^{j_{0}} t\right) \supset 4 B$. Choose also the smallest integer $j_{1}$ satisfying $B\left(x_{0}, 2^{j_{1}+2} r\right)$ $\supset B\left(u, 2^{j_{0}} t\right)$. Notice that $j_{0} \geq j_{1}$. Then, we get

$$
\begin{aligned}
& \left|f_{B(u, t)}-f_{4 B}\right| \leq \sum_{j=1}^{j_{0}}\left|f_{B\left(u, 2^{j-1} t\right)}-f_{B\left(u, 2^{j} t\right)}\right|+\left|f_{B\left(u, 2^{j} 0 t\right)}-f_{B\left(x_{0}, 2^{j_{1}+2} r\right)}\right| \\
& \quad+\sum_{j=1}^{j_{1}}\left|f_{B\left(x_{0}, 2^{j+1} r\right)}-f_{B\left(x_{0}, 2^{j+2} r\right)}\right| \\
& \leq C\left(j_{0}+1+\left(j_{1}+2\right)\right)\|f\|_{\mathrm{BMO}} \leq C\|f\|_{\mathrm{BMO}}\left(1+\log \frac{\left|u-x_{0}\right|+4 r}{t}\right) .
\end{aligned}
$$

Choose positive numbers $\gamma$ and $q_{0}$ such that $2 \gamma<\lambda n-n$ and $1<q_{0} \leq q$, and $(n-\sigma) q_{0}<n$. Then, noting $1+\log \frac{\left|u-x_{0}\right|+4 r}{t} \leq 1+C \log \frac{|u-x|}{t} \leq C\left(\frac{|u-x|}{t}\right)^{\gamma}$ for $|u-x|>2 r>2 t$, we have

$$
\begin{aligned}
& \left|\int_{|y-u| \leq t} \frac{\Omega(u-y) f_{3}(y)}{|u-y|^{n-\rho}} d y\right| \\
& \leq\left(\int_{|y-u| \leq t} \frac{|\Omega(u-y)|^{q_{0}} d y}{|u-y|^{(n-\sigma) q_{0}}}\right)^{1 / q_{0}}\left(\int_{|y-u| \leq t}\left|f(y)-f_{4 B}\right|^{q_{0}^{\prime}} \chi_{(4 B)^{c}} d y\right)^{1 / q_{0}^{\prime}} \\
& \leq C\|\Omega\|_{L^{q_{0}\left(S^{n-1}\right)}} t^{\sigma-n+\frac{n}{q_{0}}}\left\{\left(\int_{|y-u|<t}\left|f(y)-f_{B(u, t)}\right|^{q_{0}^{\prime}} d y\right)^{1 / q_{0}^{\prime}}\right. \\
& \left.+\left|f_{B(u, t)}-f_{4 B}\right|\left(\int_{|y-u|<t} d y\right)^{1 / q_{0}^{\prime}}\right\} \\
& \leq C\|\Omega\|_{L^{q}\left(S^{n-1}\right)} t^{\sigma}\left(\frac{|u-x|}{t}\right)^{\gamma}\|f\|_{\mathrm{BMO}} .
\end{aligned}
$$

Therefore

$\mu_{\lambda, 0}^{*, \rho}\left(f_{3}\right)(x) \leq C\|\Omega\|_{L^{q}\left(S^{n-1}\right)}\|f\|_{\mathrm{BMO}}\left(\int_{0}^{r} \int_{|u-x|>2 r}\left(\frac{t}{|u-x|}\right)^{\lambda n-2 \gamma} \frac{d u d t}{t^{n+1}}\right)^{1 / 2}$ 


$$
\begin{aligned}
& \leq C\|\Omega\|_{L^{q}\left(S^{n-1}\right)}\|f\|_{\mathrm{BMO}}\left(\int_{0}^{r} t^{\lambda n-2 \gamma-n-1} d t \int_{|u-x|>2 r} \frac{d u}{|u-x|^{\lambda n-2 \gamma}}\right)^{1 / 2} \\
& \leq C\|\Omega\|_{L^{q}\left(S^{n-1}\right)}\|f\|_{\mathrm{BMO}} .
\end{aligned}
$$

Proof of (c). Since $0<\alpha<1, \mathcal{E}^{\alpha, p}=\operatorname{Lip}_{\alpha}(1 \leq p<\infty)$, and the norms are equivalent. For $y \in(4 B)^{c}, x \in B, z \in 4 B$ and $u \in \mathbb{R}^{n}$, we have $|y-z| \leq|y-x|+\left|x-x_{0}\right|+\left|x_{0}-z\right| \leq|y-x|+5 r$ and $|y-x| \geq\left|y-x_{0}\right|-\left|x_{0}-x\right| \geq$ $4 r-r=3 r$, and hence $|y-z| \leq \frac{8}{3}|y-x| \leq \frac{8}{3}(|y-u|+|u-x|)$.

Thus, we have

$$
\left|f_{3}(y)\right| \leq \frac{1}{|4 B|} \int_{4 B}|f(y)-f(z)| d z \leq C\|f\|_{\operatorname{Lip}_{\alpha}}(|y-u|+|u-x|)^{\alpha},
$$

and hence

$$
\begin{aligned}
& \left|\int_{|y-u| \leq t} \frac{\Omega(u-y) f_{3}(y)}{|u-y|^{n-\rho}} d y\right| \\
& \quad \leq C\|f\|_{\operatorname{Lip}_{\alpha}} \int_{|y-u| \leq t} \frac{|\Omega(u-y)|(|y-u|+|u-x|)^{\alpha} d y}{|u-y|^{n-\sigma}} \\
& \quad \leq C\|\Omega\|_{L^{1}\left(S^{n-1}\right)}\|f\|_{\operatorname{Lip}_{\alpha}} \int_{0}^{t}(s+|u-x|)^{\alpha} s^{\sigma-1} d s \\
& \quad \leq C\|\Omega\|_{L^{1}\left(S^{n-1}\right)}\|f\|_{\operatorname{Lip}_{\alpha}}(t+|u-x|)^{\alpha} t^{\sigma} .
\end{aligned}
$$

Thus, noting $\lambda n-2 \alpha>n$ and $\alpha>0$ we have

$$
\begin{aligned}
\mu_{\lambda, 0}^{*, \rho}\left(f_{3}\right)(x) & \leq C\left(\int_{0}^{r}\left(\int_{\mathbb{R}^{n}}(t+|u-x|)^{2 \alpha}\left(\frac{t}{t+|u-x|}\right)^{\lambda n} \frac{d u d t}{t^{n+1}}\right)^{1 / 2}\right. \\
& \leq C\left(\int_{0}^{r}\left(\int_{\mathbb{R}^{n}}\left(\frac{1}{1+\frac{|u-x|}{t}}\right)^{\lambda n-2 \alpha} \frac{d u}{t^{n}} t^{2 \alpha-1} d t\right)^{1 / 2}\right. \\
& \leq C r^{\alpha}\|\Omega\|_{L^{1}\left(S^{n-1}\right)}\|f\|_{\operatorname{Lip}_{\alpha}} .
\end{aligned}
$$

Lemma 2.10. Let $1 \leq p<\infty$. Let $\Omega \in L^{1}\left(S^{n-1}\right), \rho=\sigma+i \tau(\sigma>$ $0, \tau \in \mathbb{R}), \lambda>1$, and $0<\alpha<1$. Then, for any $f \in \mathcal{E}^{\alpha, p}$, for any ball $B=B\left(x_{0}, r\right)$ and any $x \in B$

$$
\mu_{\lambda, 0,0}^{*, \rho}\left(f_{3}\right)(x) \leq C r^{\alpha}\|\Omega\|_{L^{1}\left(S^{n-1}\right)}\|f\|_{\mathcal{E}^{\alpha, p}}
$$


Proof. In this case, $\mathcal{E}^{\alpha, p}=\operatorname{Lip}_{\alpha}(1 \leq p<\infty)$, and the norms are equivalent. So, for $y \in(4 B)^{c}$ we have $\left|f_{3}(y)\right|=\left|f(y)-f_{4 B}\right| \leq \mid f(y)-$ $f\left(x_{0}\right)|+| f\left(x_{0}\right)-f_{4 B}\left|\leq\|f\|_{\operatorname{Lip}_{\alpha}}\left(\left|y-x_{0}\right|^{\alpha}+r^{\alpha}\right) \leq C\|f\|_{\operatorname{Lip}_{\alpha}}\right| y-\left.x_{0}\right|^{\alpha}$. For $\left|x-x_{0}\right| \leq r,|u-y| \leq t \leq r$ and $|u-x| \leq 8 r$, we have $\left|y-x_{0}\right| \leq$ $|y-u|+|u-x|+\left|x-x_{0}\right| \leq 10 r$, and for $\left|x-x_{0}\right| \leq r,|u-y| \leq t \leq r$ and $y \in(4 B)^{c}$ we have $|u-x| \geq\left|y-x_{0}\right|-|u-y|-\left|x_{0}-x\right|>\frac{1}{2}\left|y-x_{0}\right|>2 r$. Hence we have

$$
\begin{aligned}
& \mu_{\lambda, 0,0}^{*, \rho}\left(f_{3}\right)(x) \\
& \leq C\left(\int_{0}^{r} \int_{2 r<|u-x| \leq 8 r}\left|\frac{1}{t^{\sigma}} \int_{\substack{4 r<\left|y-x_{0}\right| \leq 10 r \\
|y-u| \leq t}} \frac{|\Omega(u-y)|\left|y-x_{0}\right|^{\alpha}}{|u-y|^{n-\sigma}} d y\right|^{2}\right. \\
& \left.\quad \times\left(\frac{t}{t+2 r}\right)^{\lambda n} \frac{d u d t}{t^{n+1}}\right)^{1 / 2}\|f\|_{\operatorname{Lip}_{\alpha}} \\
& \leq C\left(\int_{0}^{r} \int_{2 r<|u-x| \leq 8 r}\left|\frac{1}{t^{\sigma}} \int_{|y-u| \leq t} \frac{|\Omega(u-y)| r^{\alpha}}{|u-y|^{n-\sigma}} d y\right|^{2} t^{\lambda n} r^{-\lambda n} \frac{d u d t}{t^{n+1}}\right)^{1 / 2} \\
& \leq C\left(\int_{0}^{r} r^{2 \alpha-\lambda n} r^{n} t^{\lambda n-n-1} d t\right)^{1 / 2}\|\Omega\|_{L^{1}\left(S^{n-1}\right)}\|f\|_{\operatorname{Lip}_{\alpha}} \\
& \leq C r^{\alpha}\|\Omega\|_{L^{1}\left(S^{n-1}\right)}\|f\|_{\operatorname{Lip}_{\alpha} .}
\end{aligned}
$$

LEMMA 2.11. Suppose one of the following five conditions is satisfied:

(i) $\sigma>0, \lambda>1, \alpha=0,1 \leq p<\infty$. Moreover, $\Omega \in L^{q}\left(S^{n-1}\right)$ for some $q>1$ and satisfies the cancellation condition.

(ii) $\sigma>0,0<\beta \leq 1,0<\alpha<\min \{1 / 2, \min \{\beta, \sigma\}\}, 1 \leq p<\infty$, and $\lambda>1$. Moreover, $\Omega \in L^{1}\left(S^{n-1}\right)$ and satisfies the cancellation condition.

(iii) $\sigma>0,0<\beta \leq 1,1 / 2 \leq \alpha<\min \{\beta, \sigma\}, 1 \leq p<\infty$, and $\lambda>1+2 \alpha / n$. Moreover, $\Omega \in L^{1}\left(S^{n-1}\right)$ and satisfies $L^{1}-\beta$-Dini condition and the cancellation condition.

(iv) $\max \left\{1, \frac{2 n}{n+2 \sigma}\right\}<p<+\infty,-n / p<\alpha<0, \sigma>-\alpha$ and $\lambda>1$. Moreover, $\Omega \in L^{p_{0}^{\prime}}\left(S^{n-1}\right)$ and satisfies the cancellation condition, where $p_{0}=\max \{2, p\}$.

(v) $1<p<\infty,-n / p \leq \alpha<0, \sigma>n / 2$, and $\lambda>2$. Moreover, $\Omega \in L^{2}\left(S^{n-1}\right)$ and satisfies $L^{2}-\log \beta$ Dini condition for some $\beta>1$ and the cancellation condition. 
Then there exists $C>0$ such that for any ball $B=B\left(x_{0}, r\right)$ and any $f \in \mathcal{E}^{\alpha, p}$ satisfying $\mu_{\lambda, \infty}^{*, \rho}\left(f_{3}\right)\left(x_{0}\right)<+\infty$, it holds

$$
\mu_{\lambda, \infty}^{*, \rho}\left(f_{3}\right)(x)<+\infty \quad \text { and } \quad\left|\mu_{\lambda, \infty}^{*, \rho}\left(f_{3}\right)(x)-\mu_{\lambda, \infty}^{*, \rho}\left(f_{3}\right)\left(x_{0}\right)\right| \leq C r^{\alpha}\|f\|_{\mathcal{E}^{\alpha, p}}
$$

for any $x \in B$, where $f_{3}(x)=\left(f(x)-f_{4 B}\right) \chi_{(4 B)^{c}}$.

Proof. Let $x \in B$. Then, we see easily

$$
\begin{gathered}
\left|\mu_{\lambda, \infty}^{*, \rho}\left(f_{3}\right)(x)-\mu_{\lambda, \infty}^{*, \rho}\left(f_{3}\right)\left(x_{0}\right)\right| \\
\leq\left(\int_{r}^{\infty} \int_{\mathbb{R}^{n}}\left|\frac{1}{t^{\rho}} \int_{|y-u| \leq t} \frac{\Omega(u-y) f_{3}(y)}{|u-y|^{n-\rho}} d y\right|^{2}\right. \\
\left.\quad \times\left|\left(1+\frac{|u-x|}{t}\right)^{-\lambda n}-\left(1+\frac{\left|u-x_{0}\right|}{t}\right)^{-\lambda n}\right| \frac{d u d t}{t^{n+1}}\right)^{1 / 2} \\
\leq\left(\int_{r}^{\infty} \int_{\left|u-x_{0}\right|>8 r} \int_{|y-u| \leq t} \frac{\Omega(u-y) f_{3}(y)}{\left.|u-y|^{n-\rho} d y\right|^{2}}\right. \\
\left.\quad \times\left|\left(1+\frac{|u-x|}{t}\right)^{-\lambda n}-\left(1+\frac{\left|u-x_{0}\right|}{t}\right)^{-\lambda n}\right| \frac{d u d t}{t^{n+1}}\right)^{1 / 2} \\
\quad\left(\int_{r}^{\infty} \int_{\left|u-x_{0}\right| \leq 8 r} \int_{|y-u| \leq t} \frac{\Omega(u-y) f_{3}(y)}{\left.|u-y|^{n-\rho} d y\right|^{2}}\right. \\
\left.\times\left|\left(1+\frac{|u-x|}{t}\right)^{-\lambda n}-\left(1+\frac{\left|u-x_{0}\right|}{t}\right)^{-\lambda n}\right| \frac{d u d t}{t^{n+1}}\right)^{1 / 2}
\end{gathered}
$$

$=: I_{1}+I_{2}$.

(i) In this case, $\mathcal{E}^{0, s}=\mathrm{BMO}(1 \leq s<\infty)$, and the norms are equivalent. Firstly we note that for $\alpha=0$

$$
\left(\int_{\left|y-x_{0}\right| \leq R}\left|f_{3}(y)\right|^{s} d y\right)^{1 / s} \leq C\|f\|_{\mathrm{BMO}} R^{n / s} \log \frac{R}{r} \quad(R>4 r)
$$

This can be seen like as in Lemma 2.3. Choose $q_{0}>1$ such that $q_{0}^{\prime}>$ $\max \left\{q^{\prime}, \frac{n}{\sigma}, \frac{2}{\lambda-1+\frac{1}{n}}\right\}$. Then, since $\left|y-x_{0}\right| \leq|y-u|+\left|u-x_{0}\right| \leq t+\left|u-x_{0}\right|$ 
for $|y-u|<t$, we get by using the Hölder inequality

$$
\begin{aligned}
& \left|\int_{|y-u| \leq t} \frac{\Omega(u-y) f_{3}(y)}{|u-y|^{n-\rho}} d y\right| \\
& \quad \leq\left(\int_{|y-u| \leq t} \frac{|\Omega(u-y)|^{q_{0}}}{|u-y|^{q_{0}(n-\sigma)}} d y\right)^{1 / q_{0}}\left(\int_{\left|y-x_{0}\right| \leq t+\left|u-x_{0}\right|}\left|f_{3}(y)\right|^{q_{0}^{\prime}} d y\right)^{1 / q_{0}^{\prime}} \\
& \quad \leq C\|\Omega\|_{L^{q_{0}\left(S^{n-1}\right)}} t^{\sigma-n+n / q_{0}}\|f\|_{\mathrm{BMO}}\left(t+\left|u-x_{0}\right|\right)^{n / q_{0}^{\prime}} \log \frac{\left(t+\left|u-x_{0}\right|\right)}{r} .
\end{aligned}
$$

By the mean value theorem, we get

$$
\left|\left(1+\frac{|u-x|}{t}\right)^{-\lambda n}-\left(1+\frac{\left|u-x_{0}\right|}{t}\right)^{-\lambda n}\right| \leq C \frac{\left|x-x_{0}\right|}{t}\left(1+\frac{\left|u-x_{0}\right|}{t}\right)^{-\lambda n-1},
$$

for $\left|u-x_{0}\right|>8 r$ and $x \in B$. Hence, we have

$$
\begin{aligned}
& I_{1} \leq C\|\Omega\|_{L^{q_{0}\left(S^{n-1}\right)}}\|f\|_{\mathrm{BMO}}\left(\int_{r}^{\infty} \int_{\left|u-x_{0}\right|>8 r} t^{-2 n / q_{0}^{\prime}}\left(t+\left|u-x_{0}\right|\right)^{2 n / q_{0}^{\prime}}\right. \\
& \left.\quad \times \log ^{2} \frac{\left(t+\left|u-x_{0}\right|\right)}{r} \frac{r}{t}\left(1+\frac{\left|u-x_{0}\right|}{t}\right)^{-\lambda n-1} \frac{d u d t}{t^{n+1}}\right)^{1 / 2} \\
& \leq C\|\Omega\|_{L^{q_{0}\left(S^{n-1}\right)}}\|f\|_{\mathrm{BMO}}\left(\int _ { r } ^ { \infty } r \left(\int_{\mathbb{R}^{n}}\left(1+\frac{\left|u-x_{0}\right|}{t}\right)^{-\lambda n-1+2 n / q_{0}^{\prime}}\right.\right. \\
& \left.\left.\quad \times \log ^{2} \frac{\left(t+\left|u-x_{0}\right|\right)}{r} \frac{d u}{t^{n}}\right) \frac{d t}{t^{2}}\right)^{1 / 2} \\
& \leq C\|\Omega\|_{L^{q_{0}\left(S^{n-1}\right)}}\|f\|_{\mathrm{BMO}}\left(\int_{r}^{\infty} r\left(\log ^{2} \frac{t}{r}+1\right) \frac{d t}{t^{2}}\right)^{1 / 2} \\
& \leq C\|\Omega\|_{L^{q}\left(S^{n-1}\right)}\|f\|_{\mathrm{BMO}} .
\end{aligned}
$$

As for $I_{2}$, since $t+\left|u-x_{0}\right| \leq 9 t$ for $\left|u-x_{0}\right| \leq 8 r$ and $t>r$, we have, using the mean value theorem,

$$
\left|\left(1+\frac{|u-x|}{t}\right)^{-\lambda n}-\left(1+\frac{\left|u-x_{0}\right|}{t}\right)^{-\lambda n}\right| \leq C \frac{\left|x-x_{0}\right|}{t} .
$$

Hence, we obtain

$$
\begin{aligned}
I_{2} & \leq C\|\Omega\|_{L^{q_{0}\left(S^{n-1}\right)}}\|f\|_{\mathrm{BMO}}\left(\int_{r}^{\infty} \int_{\left|u-x_{0}\right| \leq 8 r} t^{-2 n / q_{0}^{\prime}} t^{2 n / q_{0}^{\prime}} \frac{r}{t} \log ^{2} \frac{t}{r} \frac{d u d t}{t^{n+1}}\right)^{1 / 2} \\
& \leq C\|\Omega\|_{L^{q}\left(S^{n-1}\right)}\|f\|_{\mathrm{BMO}} .
\end{aligned}
$$


Thus, we obtain the desired estimate in the case (i).

(ii) In this case, $0<\alpha<1 / 2$, and $\mathcal{E}^{\alpha, p}=\operatorname{Lip}_{\alpha}(1 \leq p<\infty)$, and the norms are equivalent. Let $x \in B$.

For $t>r, y \in(4 B)^{c},|y-u|<t, z \in 4 B$ and $u \in \mathbb{R}^{n}$, we have $|y-z| \leq|y-u|+\left|u-x_{0}\right|+\left|x_{0}-z\right| \leq C\left(t+\left|u-x_{0}\right|\right)$, and hence $\left|f_{3}(y)\right| \leq$ $C\|f\|_{\operatorname{Lip}_{\alpha}}\left(t+\left|u-x_{0}\right|\right)^{\alpha}$. So, we have

$$
\left|\int_{|y-u| \leq t} \frac{\Omega(u-y) f_{3}(y)}{|u-y|^{n-\rho}} d y\right| \leq C\|\Omega\|_{L^{1}\left(S^{n-1}\right)}\|f\|_{\operatorname{Lip}_{\alpha}}\left(t+\left|u-x_{0}\right|\right)^{\alpha} t^{\sigma} .
$$

We get, as in the estimate for $I_{1}$ in the case (i),

$$
\begin{aligned}
& I_{1} \leq C\left(\int_{r}^{\infty} \int_{\left|u-x_{0}\right|>8 r}\left|\frac{1}{t^{\rho}} \int_{|y-u| \leq t} \frac{\Omega(u-y) f_{3}(y)}{|u-y|^{n-\rho}} d y\right|^{2}\right. \\
&\left.\times \frac{\left|x-x_{0}\right|}{t}\left(1+\frac{\left|u-x_{0}\right|}{t}\right)^{-\lambda n-1} \frac{d u d t}{t^{n+1}}\right)^{1 / 2} \\
& \leq C\|\Omega\|_{L^{1}\left(S^{n-1}\right)}\|f\|_{\operatorname{Lip}_{\alpha}} r^{1 / 2} \\
& \times\left(\int_{r}^{\infty} \int_{\left|u-x_{0}\right|>8 r}\left(1+\frac{\left|u-x_{0}\right|}{t}\right)^{-\lambda n-1+2 \alpha} \frac{d u d t}{t^{n+2-2 \alpha}}\right)^{1 / 2} \\
& \leq C\|\Omega\|_{L^{1}\left(S^{n-1}\right)}\|f\|_{\operatorname{Lip}_{\alpha}} r^{1 / 2} \\
& \times\left(\int_{r}^{\infty}\left(\int_{\mathbb{R}^{n}}\left(1+\frac{\left|u-x_{0}\right|}{t}\right)^{-\lambda n-1+2 \alpha} \frac{d u}{t^{n}}\right) \frac{d t}{t^{2-2 \alpha}}\right)^{1 / 2} \\
& \leq C\|\Omega\|_{L^{1}\left(S^{n-1}\right)}\|f\|_{\operatorname{Lip}_{\alpha}} r^{\alpha} .
\end{aligned}
$$

In the above, we have used $\alpha<1 / 2$.

Next, for $\left|u-x_{0}\right| \leq 8 r$ and $t>r$, we have $t+\left|u-x_{0}\right| \leq 9 t$, and so, $\left|f_{3}(y)\right| \leq C\|f\|_{\operatorname{Lip}_{\alpha}} t^{\alpha}$. Hence, we obtain, as in the estimate for $I_{2}$ in the case (i),

$$
\begin{aligned}
I_{2} \leq C & \|f\|_{\operatorname{Lip}_{\alpha}} \\
& \times\left(\int_{r}^{\infty} \int_{\left|u-x_{0}\right| \leq 8 r}\left|\frac{1}{t^{\sigma}} \int_{|y-u| \leq t} \frac{|\Omega(u-y)| t^{\alpha}}{|u-y|^{n-\sigma}} d y\right|^{2} \frac{\left|x-x_{0}\right|}{t} \frac{d u d t}{t^{n+1}}\right)^{1 / 2} \\
\leq & C\|\Omega\|_{L^{1}\left(S^{n-1}\right)}\|f\|_{\operatorname{Lip}_{\alpha}} r^{1 / 2}\left(\int_{r}^{\infty} \int_{\left|u-x_{0}\right| \leq 8 r} t^{2 \alpha} \frac{d u d t}{t^{n+2}}\right)^{1 / 2} \\
\leq & C\|\Omega\|_{L^{1}\left(S^{n-1}\right)}\|f\|_{\operatorname{Lip}_{\alpha}} r^{\alpha} .
\end{aligned}
$$


Thus, summing the estimates for $I_{1}$ and $I_{2}$, we have $\left|\mu_{\lambda, \infty}^{*, \rho}\left(f_{3}\right)(x)-\mu_{\lambda, \infty}^{*, \rho}\left(f_{3}\right)\left(x_{0}\right)\right| \leq C r^{\alpha}\|\Omega\|_{L^{1}\left(S^{n-1}\right)}\|f\|_{\mathcal{E}^{\alpha, p}} \quad$ for any $x \in B$

(iii) Also, in this case, $\mathcal{E}^{\alpha, p}=\operatorname{Lip}_{\alpha}(1 \leq p<\infty)$, and the norms are equivalent. Let $x \in B$. Using the expression

$$
\begin{aligned}
& \mu_{\lambda, \infty}^{*, \rho}\left(f_{3}\right)(x) \\
& =\left(\int_{r}^{\infty} \int_{\mathbb{R}^{n}}\left|\frac{1}{t^{\rho}} \int_{|x-u-y| \leq t} \frac{\Omega(x-u-y) f_{3}(y)}{|x-u-y|^{n-\rho}} d y\right|^{2}\left(\frac{t}{t+|u|}\right)^{\lambda n} \frac{d u d t}{t^{n+1}}\right)^{1 / 2},
\end{aligned}
$$

we see that

$$
\begin{aligned}
& \left|\mu_{\lambda, \infty}^{*, \rho}\left(f_{3}\right)(x)-\mu_{\lambda, \infty}^{*, \rho}\left(f_{3}\right)\left(x_{0}\right)\right| \\
& \leq\left(\int_{r}^{\infty} \int_{\mathbb{R}^{n}} \mid \frac{1}{t^{\rho}} \int_{|x-u-y| \leq t} \frac{\Omega(x-u-y) f_{3}(y)}{|x-u-y|^{n-\rho}} d y\right. \\
& \left.-\left.\frac{1}{t^{\rho}} \int_{\left|x_{0}-u-y\right| \leq t} \frac{\Omega\left(x_{0}-u-y\right) f_{3}(y)}{\left|x_{0}-u-y\right|^{n-\rho}} d y\right|^{2}\left(1+\frac{|u|}{t}\right)^{-\lambda n} \frac{d u d t}{t^{n+1}}\right)^{1 / 2} \\
& \leq\left(\int_{r}^{\infty} \int_{\mathbb{R}^{n}}\left|\frac{1}{t^{\rho}} \int_{\substack{|x-u-y| \leq t \\
\left|x_{0}-u-y\right|>t}} \frac{\Omega(x-u-y) f_{3}(y)}{|x-u-y|^{n-\rho}} d y\right|^{2}\left(1+\frac{|u|}{t}\right)^{-\lambda n} \frac{d u d t}{t^{n+1}}\right)^{1 / 2} \\
& +\left(\int_{r}^{\infty} \int_{\mathbb{R}^{n}}\left|\frac{1}{t^{\rho}} \int_{\substack{\left|x_{0}-u-y\right| \leq t \\
|x-u-y|>t}} \frac{\Omega(x-u-y) f_{3}(y)}{\left|x_{0}-u-y\right|^{n-\rho}} d y\right|^{2}\left(1+\frac{|u|}{t}\right)^{-\lambda n} \frac{d u d t}{t^{n+1}}\right)^{1 / 2} \\
& +\left(\int_{r}^{\infty} \int_{\mathbb{R}^{n}}\left|\frac{1}{t^{\rho}} \int_{\substack{\left|x_{0}-u-y\right| \leq t \\
|x-u-y| \leq t}}\left(\frac{\Omega(x-u-y)}{|x-u-y|^{n-\rho}}-\frac{\Omega\left(x_{0}-u-y\right)}{\left|x_{0}-u-y\right|^{n-\rho}}\right) f_{3}(y) d y\right|^{2}\right. \\
& \left.\times\left(1+\frac{|u|}{t}\right)^{-\lambda n} \frac{d u d t}{t^{n+1}}\right)^{1 / 2} \\
& =: L_{1}+L_{2}+L_{3} \text {. }
\end{aligned}
$$

For $y \in(4 B)^{c}, z \in 4 B$, and $u \in \mathbb{R}^{n}$, we have $|y-z| \leq|y-x+u|+|u|+|x-z| \leq$ $|y-x+u|+|u|+5 r$, and hence $\left|f_{3}(y)\right| \leq C\|f\|_{\operatorname{Lip}_{\alpha}}(|y-x+u|+|u|+r)^{\alpha}$. Thus for $L_{1}$, we have using Lemma 1.3

$$
\begin{aligned}
& \left|\frac{1}{t^{\rho}} \int_{\substack{|x-u-y| \leq t \\
\left|x_{0}-u-y\right|>t}} \frac{\Omega(x-u-y) f_{3}(y)}{|x-u-y|^{n-\rho}} d y\right| \\
& \leq C\|f\|_{\operatorname{Lip}_{\alpha}} t^{-\sigma} \int_{\substack{|x-u-y| \leq t \\
\left|x_{0}-u-y\right|>t}} \frac{|\Omega(x-u-y)|\left(|y-x+u|^{\alpha}+|u|^{\alpha}+r^{\alpha}\right)}{|x-u-y|^{n-\sigma}} d y
\end{aligned}
$$




$$
\begin{aligned}
& \leq C\|f\|_{\operatorname{Lip}_{\alpha}} t^{-\sigma}\|\Omega\|_{L^{1}\left(S^{n-1}\right)} \int_{t-r}^{t} s^{\sigma-1}\left(s^{\alpha}+|u|^{\alpha}+r^{\alpha}\right) d s \\
& \leq C\|f\|_{\operatorname{Lip}_{\alpha}}\|\Omega\|_{L^{1}\left(S^{n-1}\right)} t^{-\sigma} r t^{\sigma-1}\left(t^{\alpha}+|u|^{\alpha}+r^{\alpha}\right) \\
& \leq C\|f\|_{\operatorname{Lip}_{\alpha}}\|\Omega\|_{L^{1}\left(S^{n-1}\right)} r t^{-1}\left(t^{\alpha}+|u|^{\alpha}\right) .
\end{aligned}
$$

Hence we obtain

$$
\begin{aligned}
L_{1} \leq C\|f\|_{\operatorname{Lip}_{\alpha}}\|\Omega\|_{L^{1}\left(S^{n-1}\right)} & \\
& \times\left(\int_{r}^{\infty} \int_{\mathbb{R}^{n}} r^{2} t^{-2}\left(t^{2 \alpha}+|u|^{2 \alpha}\right)\left(1+\frac{|u|}{t}\right)^{-\lambda n} \frac{d u d t}{t^{n+1}}\right)^{1 / 2} \\
& \leq C\|f\|_{\operatorname{Lip}_{\alpha}}\|\Omega\|_{L^{1}\left(S^{n-1}\right)} r\left(\int_{r}^{\infty}\left(\int_{\mathbb{R}^{n}}\left(1+\frac{|u|}{t}\right)^{-\lambda n+2 \alpha} \frac{d u}{t^{n}}\right) \frac{d t}{t^{3-2 \alpha}}\right)^{1 / 2} \\
& \leq C\|f\|_{\operatorname{Lip}_{\alpha}}\|\Omega\|_{L^{1}\left(S^{n-1}\right)} r^{\alpha} .
\end{aligned}
$$

Similarly, we obtain the same estimate for $L_{2}$. We turn to the estimate for $L_{3}$. Since $\left\{\left|x_{0}-u-y\right| \leq t,|x-u-y| \leq t\right\} \subset\left\{4 r<\left|x_{0}-u-y\right| \leq\right.$ $2 t\} \cup\left\{\left|x_{0}-u-y\right| \leq 4 r\right\}$ and $\left\{\left|x_{0}-u-y\right| \leq 4 r\right\} \subset\{|x-u-y| \leq 5 r\}$ for $x \in B$, we see

$$
\begin{aligned}
L_{3} \leq & \left(\int_{r}^{\infty} \int_{\mathbb{R}^{n}}\left|\frac{1}{t^{\rho}} \int_{4 r<\left|x_{0}-u-y\right| \leq 2 t}\right| \frac{\Omega(x-u-y)}{|x-u-y|^{n-\rho}}-\frac{\Omega\left(x_{0}-u-y\right)}{\left|x_{0}-u-y\right|^{n-\rho} \mid}\right. \\
& \left.\times\left.\left|f_{3}(y)\right| d y\right|^{2}\left(1+\frac{|u|}{t}\right)^{-\lambda n} \frac{d u d t}{t^{n+1}}\right)^{1 / 2} \\
& +\left(\int_{r}^{\infty} \int_{\mathbb{R}^{n}}\left|\frac{1}{t^{\rho}} \int_{\left|x_{0}-u-y\right| \leq 4 r}\right| \frac{\Omega\left(x_{0}-u-y\right)}{\left|x_{0}-u-y\right|^{n-\rho}}|| f_{3}(y)|d y|^{2}\right. \\
& \left.+\left(\int_{r}^{\infty} \int_{\mathbb{R}^{n}}\left|\frac{1}{t^{\rho}} \int_{|x-u-y| \leq 5 r}\right| \frac{\Omega(x \mid}{t}\right)^{-\lambda n} \frac{d u d t}{t^{n+1}}\right)^{1 / 2} \\
& \left.\times\left(1+\frac{|u|}{t}\right)^{-\lambda n} \frac{d u d t}{t^{n+1}}\right)^{1 / 2} \\
=: & L_{31}+L_{32}+L_{33} .
\end{aligned}
$$

In this case, we have as before $\left|f_{3}(y)\right| \leq C\|f\|_{\operatorname{Lip}_{\alpha}}(|y-x+u|+|u|+r)^{\alpha} \leq$ 
$C\|f\|_{\operatorname{Lip}_{\alpha}}(t+|u|)^{\alpha}$. Hence, if $\sigma>1$, as in the proof of Lemma 2.3, we have

$$
\begin{aligned}
& \left|\frac{1}{t^{\rho}} \int_{4 r<\left|x_{0}-u-y\right| \leq 2 t}\right| \frac{\Omega(x-u-y)}{|x-u-y|^{n-\rho}}-\frac{\Omega\left(x_{0}-u-y\right)}{\left|x_{0}-u-y\right|^{n-\rho}}|| f_{3}(y)|d y| \\
& \leq C\|f\|_{\operatorname{Lip}_{\alpha}}(t+|u|)^{\alpha} t^{-\sigma} \\
& \quad \times \int_{4 r<\left|x_{0}-u-y\right| \leq 2 t}\left|\frac{\Omega(x-u-y)}{|x-u-y|^{n-\rho}}-\frac{\Omega\left(x_{0}-u-y\right)}{\left|x_{0}-u-y\right|^{n-\rho}}\right| d y \\
& \leq C\|f\|_{\operatorname{Lip}_{\alpha}}(t+|u|)^{\alpha} t^{-\sigma} r^{\sigma}\left(\left(\frac{t}{r}\right)^{\sigma-1}+\left(\frac{t}{r}\right)^{\sigma-\beta}\right) \\
& \leq C\|f\|_{\operatorname{Lip}_{\alpha}}(1+|u| / t)^{\alpha} t^{\alpha}\left(\frac{r}{t}+\left(\frac{r}{t}\right)^{\beta}\right) .
\end{aligned}
$$

Thus, noting $\beta>\alpha$ and $\lambda n>n+2 \alpha$, we have

$$
\begin{aligned}
L_{31} & \leq C\|f\|_{\operatorname{Lip}_{\alpha}}\left(\int_{r}^{\infty}\left(\int_{\mathbb{R}^{n}}\left(1+\frac{|u|}{t}\right)^{-\lambda n+2 \alpha} \frac{d u}{t^{n}}\right)\left(\frac{r^{2}}{t^{2}}+\frac{r^{2 \beta}}{t^{2 \beta}}\right) \frac{d t}{t^{1-2 \alpha}}\right)^{1 / 2} \\
& \leq C\|f\|_{\operatorname{Lip}_{\alpha}}\left(r^{2} r^{2 \alpha-2}+r^{2 \beta} r^{2 \alpha-2 \beta}\right)^{1 / 2} \leq C\|f\|_{\operatorname{Lip}_{\alpha}} r^{\alpha} .
\end{aligned}
$$

The other three cases $\sigma=1,0<\beta<\sigma<1$, and $0<\sigma<\beta \leq 1$ and $\sigma<1$ are treated in the same way as in the corresponding cases in Lemma 2.3, respectively.

As for $L_{32}$, we have as above $\left|f_{3}(y)\right| \leq C\|f\|_{\operatorname{Lip}_{\alpha}}(t+|u|)^{\alpha}$. Using this and noting $\alpha<\sigma$ and $\lambda n>n+2 \alpha$, we get

$$
\begin{aligned}
& L_{32} \leq C\|f\|_{\operatorname{Lip}_{\alpha}}\left(\int_{r}^{\infty} \int_{\mathbb{R}^{n}}\left|\frac{1}{t^{\rho}} \int_{\left|x_{0}-u-y\right| \leq 4 r}\right| \frac{\Omega\left(x_{0}-u-y\right)}{\left|x_{0}-u-y\right|^{n-\rho}}\left|(t+|u|)^{\alpha} d y\right|^{2}\right. \\
& \left.\times\left(1+\frac{|u|}{t}\right)^{-\lambda n} \frac{d u d t}{t^{n+1}}\right)^{1 / 2} \\
& \leq C\|f\|_{\operatorname{Lip}_{\alpha}}\|\Omega\|_{L^{1}\left(S^{n-1}\right)}\left(\int_{r}^{\infty} \int_{\mathbb{R}^{n}} t^{-2 \sigma} r^{2 \sigma}(t+|u|)^{2 \alpha}\left(1+\frac{|u|}{t}\right)^{-\lambda n} \frac{d u d t}{t^{n+1}}\right)^{1 / 2} \\
& \leq C\|f\|_{\operatorname{Lip}_{\alpha}}\|\Omega\|_{L^{1}\left(S^{n-1}\right)} r^{\sigma}\left(\int_{r}^{\infty}\left(\int_{\mathbb{R}^{n}}\left(1+\frac{|u|}{t}\right)^{-\lambda n+2 \alpha} \frac{d u}{t^{n}}\right) \frac{d t}{t^{1+2 \sigma-2 \alpha}}\right)^{1 / 2} \\
& \leq C\|f\|_{\operatorname{Lip}_{\alpha}}\|\Omega\|_{L^{1}\left(S^{n-1}\right)} r^{\sigma} r^{-(2 \sigma-2 \alpha) / 2}=C\|f\|_{\operatorname{Lip}_{\alpha}}\|\Omega\|_{L^{1}\left(S^{n-1}\right)} r^{\alpha} .
\end{aligned}
$$

Similarly we get the same estimate for $L_{33}$. Summing up the estimates for $L_{31}, L_{32}, L_{33}$, we obtain the desired estimate for $L_{3}$. Finally summing up the estimates for $L_{1}, L_{2}, L_{3}$, we get

$$
\left|\mu_{\lambda, \infty}^{*, \rho}\left(f_{3}\right)(x)-\mu_{\lambda, \infty}^{*, \rho}\left(f_{3}\right)\left(x_{0}\right)\right| \leq C\|f\|_{\operatorname{Lip}_{\alpha}} r^{\alpha} .
$$


(iv) Using the Minkowski inequality and the cancellation property of $\Omega$ we see

$$
\begin{aligned}
\mid \mu_{\lambda, \infty}^{*, \rho}\left(f_{3}\right)(x) & -\mu_{\lambda, \infty}^{*, \rho}\left(f_{3}\right)\left(x_{0}\right) \mid \\
\leq & \left(\int_{r}^{\infty} \int_{\mathbb{R}^{n}}\left|\frac{1}{t^{\rho}} \int_{|y-u| \leq t} \frac{\Omega(u-y)\left(f(y)-f_{4 B}\right)}{|u-y|^{n-\rho}} d y\right|^{2}\right. \\
& \left.\times\left|\left(1+\frac{|u-x|}{t}\right)^{-\lambda n}-\left(1+\frac{\left|u-x_{0}\right|}{t}\right)^{-\lambda n}\right| \frac{d u d t}{t^{n+1}}\right)^{1 / 2} \\
& +\left(\int_{r}^{\infty} \int_{\mathbb{R}^{n}}\left|\frac{1}{t^{\rho}} \int_{|y-u| \leq t} \frac{\Omega(u-y) f_{2}(y)}{|u-y|^{n-\rho}} d y\right|^{2}\right. \\
\times & \left.\left.\quad \int_{r}^{\infty} \int_{\mathbb{R}^{n}} \mid \frac{1}{t^{\rho}} \int_{|y-u| \leq t} \frac{|u-x|}{t}\right)^{-\lambda n}-\left(1+\frac{\left|u-x_{0}\right|}{t}\right)^{-\lambda n} \mid \frac{d u d t}{t^{n+1}}\right)^{1 / 2} \\
\quad & \left.\times\left|\left(1+\frac{|u-x|}{t}\right)^{-\lambda n}-\left(1+\frac{\left|u-x_{0}\right|}{t}\right)^{-\lambda n}\right| \frac{d u d t}{t^{n+1}}\right)^{1 / 2} \\
& +\sqrt{2}\left(\mu_{\lambda, \infty}^{*, \rho}\left(f_{2}\right)(x)+\mu_{\lambda, \infty}^{*, \rho}\left(f_{2}\right)\left(x_{0}\right)\right) \\
=: & J_{1}+J_{2} .
\end{aligned}
$$

Now, we note that for $\alpha<0$ and non-negative integer $j$

$$
\left(\int_{|y-u| \leq t / 2^{j}}\left|f(y)-f_{B(u, t)}\right|^{p} d y\right)^{1 / p} \leq C\|f\|_{\mathcal{E}^{\alpha, p}} t^{\alpha+\frac{n}{p}} 2^{-j\left(\alpha+\frac{n}{p}\right)} .
$$

This can be seen like as in Lemma 2.3. So, choosing a non-negative number $\gamma$ satisfying $n / p-\sigma<\gamma<\alpha+n / p$, we have by Hölder's inequality

$$
\begin{aligned}
& \left|\int_{|y-u| \leq t} \frac{\Omega(u-y)\left(f(y)-f_{B(u, t)}\right)}{|u-y|^{n-\rho}} d y\right| \\
& \quad \leq\left(\int_{|y-u| \leq t} \frac{|\Omega(u-y)|^{p^{\prime}}}{|u-y|^{(n-\sigma-\gamma) p^{\prime}}} d y\right)^{1 / p^{\prime}}\left(\int_{|y-u| \leq t} \frac{\left|f(y)-f_{B(u, t)}\right|^{p}}{|u-y|^{p \gamma}} d y\right)^{1 / p} \\
& \quad \leq C\|\Omega\|_{L^{p^{\prime}\left(S^{n-1}\right)}} t^{\sigma+\gamma-n+\frac{n}{p^{\prime}}}\left(\sum_{j=0}^{\infty} \int_{\frac{t}{2^{j+1}}<|y-u| \leq \frac{t}{2^{j}}} \frac{\left|f(y)-f_{B(u, t)}\right|^{p}}{|u-y|^{p \gamma}} d y\right)^{1 / p} \\
& \quad \leq C\|\Omega\|_{L^{p^{\prime}\left(S^{n-1}\right)}} t^{\sigma+\gamma-\frac{n}{p}}\left(\sum_{j=0}^{\infty} \frac{1}{\left(\frac{t}{2^{j+1}}\right)^{p \gamma}} \int_{|y-u| \leq \frac{t}{2^{j}}}\left|f(y)-f_{B(u, t)}\right|^{p} d y\right)^{1 / p}
\end{aligned}
$$


$\leq C\|\Omega\|_{L^{p^{\prime}}\left(S^{n-1}\right)}\|f\|_{\mathcal{E}^{\alpha, p}} t^{\sigma+\alpha}$.

Hence, as in the proof of $I_{1}$ in the case (i)

$$
\begin{aligned}
& J_{1} \leq C\|\Omega\|_{L^{p^{\prime}\left(S^{n-1}\right)}}\|f\|_{\mathcal{E}^{\alpha, p}}\left(\int_{r}^{\infty} \int_{\left|u-x_{0}\right|>8 r} t^{2 \alpha} \frac{r}{t}\left(1+\frac{\left|u-x_{0}\right|}{t}\right)^{-\lambda n-1} \frac{d u d t}{t^{n+1}}\right. \\
& \left.+\int_{r}^{\infty} \int_{\left|u-x_{0}\right| \leq 8 r} t^{2 \alpha} \frac{r}{t} \frac{d u d t}{t^{n+1}}\right)^{1 / 2} \\
& \leq C\|\Omega\|_{L^{p^{\prime}}\left(S^{n-1}\right)}\|f\|_{\mathcal{E}^{\alpha, p}}\left(r \int_{r}^{\infty} \int_{\mathbb{R}^{n}}\left(1+\frac{\left|u-x_{0}\right|}{t}\right)^{-\lambda n-1} \frac{d u}{t^{n}} \frac{d t}{t^{2-2 \alpha}}\right. \\
& \left.+r \int_{r}^{\infty} C r^{n} \frac{d t}{t^{n-2 \alpha+2}}\right)^{1 / 2} \\
& \leq C\|\Omega\|_{L^{p^{\prime}\left(S^{n-1}\right)}}\|f\|_{\mathcal{E}^{\alpha, p}, r^{\alpha}} .
\end{aligned}
$$

As for $J_{2}$, we have by Lemma 2.7 (a)

$$
J_{2} \leq C\|\Omega\|_{L^{p_{0}^{\prime}\left(S^{n-1}\right)}}\|f\|_{\mathcal{E}^{\alpha, p} r^{\alpha}} .
$$

Thus, we have the desired estimate in this case (iv).

(v) We use the expression in (iii), and estimate $L_{1}, L_{2}, L_{3}$. For $\mid x-$ $u-y \mid \leq t$ and $\left|y-x_{0}\right|>4 r$, we see $t+|u| \geq|x-u-y|+|u| \geq \mid x-$ $y|\geq| x_{0}-y|-| x_{0}-x\left|\geq \frac{3}{4}\right| y-x_{0} \mid$. Choose $\eta>0$ such that $0<\eta<$ $\min \{1 / 2, \lambda / 2-n, \sigma-n / 2\}$. Then, by the Minkowski inequality, Lemma 1.3 (noting $\sigma-n / 2>0$ ) and Lemma 1.1 we have

$$
\begin{aligned}
& L_{1} \leq\left(\int_{r}^{\infty} \int_{\mathbb{R}^{n}}\left(\frac{1}{t^{\sigma}} \int_{t-r<|x-u-y| \leq t} \frac{\left|\Omega(x-u-y) f_{3}(y)\right|}{|x-u-y|^{n-\sigma}} d y\right)^{2}\right. \\
& \left.\quad \times\left(\frac{t}{t+|u|}\right)^{2 n+2 \eta} \frac{d u d t}{t^{n+1}}\right)^{1 / 2} \\
& \leq \int_{\mathbb{R}^{n}}\left(\int_{r}^{\infty} \int_{t-r<|x-u-y| \leq t} \frac{|\Omega(x-u-y)|^{2}}{|x-u-y|^{2 n-2 \sigma}}\left(\frac{t}{\left|y-x_{0}\right|}\right)^{2 n+2 \eta} \frac{d u d t}{t^{n+2 \sigma+1}}\right)^{1 / 2} \\
& \leq \int_{\mathbb{R}^{n}}\left(\int_{r}^{\infty} \int_{t-r<|x-u-y| \leq t} \frac{|\Omega(x-u-y)|^{2}}{|x-u-y|^{2 n-2 \sigma}} \frac{d u d t}{t^{2 \sigma-n-2 \eta+1}}\right)^{1 / 2} \frac{\left|f_{3}(y)\right|}{\left|y-x_{0}\right|^{n+\eta}} d y \\
& \leq C\|\Omega\|_{L^{2}\left(S^{n-1}\right)} \int_{\mathbb{R}^{n}}\left(\int_{r}^{\infty} \int_{t-r}^{t} s^{2 \sigma-n-1} d s \frac{d t}{t^{2 \sigma-n-2 \eta+1}}\right)^{1 / 2} \frac{\left|f_{3}(y)\right|}{\left|y-x_{0}\right|^{n+\eta}} d y
\end{aligned}
$$




$$
\begin{aligned}
& \leq C\|\Omega\|_{L^{2}\left(S^{n-1}\right)} \int_{\mathbb{R}^{n}}\left(\int_{r}^{\infty} r t^{2 \sigma-n-1} \frac{d t}{t^{2 \sigma-n-2 \eta+1}}\right)^{1 / 2} \frac{\left|f_{3}(y)\right|}{\left|y-x_{0}\right|^{n+\eta}} d y \\
& \leq C\|\Omega\|_{L^{2}\left(S^{n-1}\right)} r^{\eta} \int_{\mathbb{R}^{n}} \frac{\left|f_{3}(y)\right|}{\left|y-x_{0}\right|^{n+\eta}} d y \leq C\|\Omega\|_{L^{2}\left(S^{n-1}\right)}\|f\|_{\mathcal{E}^{\alpha, p} r^{\alpha}} .
\end{aligned}
$$

We have used above the fact $\|f\|_{\mathcal{E}^{\alpha, 1}} r^{\alpha} \leq\|f\|_{\mathcal{E}^{\alpha, p},} r^{\alpha}$ for $p>1$.

Similarly we have the same estimate for $L_{2}$.

Next, we estimate $L_{31}, L_{32}, L_{33}$. To estimate $L_{31}$, we choose $\gamma>0$ such that $1<\gamma<\beta$, and note that for $\left|x_{0}-u-y\right|<2 t$ and $\left|y-x_{0}\right|>4 r$, we have $t+|u| \geq\left(\left|x_{0}-u-y\right|+|u|\right) / 2 \geq\left|x_{0}-y\right| / 2 \geq 2 r$, and so

$$
\begin{aligned}
\left(\frac{t}{t+|u|}\right)^{\lambda n} & \leq C\left(\frac{t}{t+|u|}\right)^{2 n}\left(\frac{1+\log \frac{t}{r}}{1+\log \frac{t+|u|}{r}}\right)^{2 \gamma} \\
& \leq C\left(\frac{t}{\left|y-x_{0}\right|}\right)^{2 n}\left(1+\log \frac{t}{r}\right)^{2 \gamma}\left(1+\log \frac{\left|y-x_{0}\right|}{r}\right)^{-2 \gamma}
\end{aligned}
$$

(This follows from an elementary inequality $\left(\log \frac{e^{1 / \gamma} t}{r}\right) /\left(\log \frac{e^{1 / \gamma} s}{r}\right)>t^{\gamma} / s^{\gamma}$ for $0<r<t<s$ and $\gamma>0$ ).

Hence, using the Minkowski inequality we get

$$
\begin{aligned}
L_{31} \leq C \int_{\mathbb{R}^{n}} & \left(\int_{r}^{\infty} \frac{1}{t^{2 \sigma}} \int_{4 r<\left|x_{0}-u-y\right| \leq 2 t}\left|\frac{\Omega(x-u-y)}{|x-u-y|^{n-\rho}}-\frac{\Omega\left(x_{0}-u-y\right)}{\left|x_{0}-u-y\right|^{n-\rho}}\right|^{2}\right. \\
& \left.\times t^{2 n}\left(1+\log \frac{t}{r}\right)^{2 \gamma} \frac{d u d t}{t^{n+1}}\right)^{1 / 2} \frac{\left|f_{3}(y)\right|}{\left|y-x_{0}\right|^{n} \log ^{\gamma} \frac{\left|y-x_{0}\right|}{r}} d y .
\end{aligned}
$$

Now, using an elementary inequality $\int_{A}^{\infty} \frac{\log ^{b}}{s^{1+a}} d s \leq C \frac{\log ^{b} A}{A^{a}}$ for $A \geq 1, a, b>$ 0 (This can be seen by integrating by parts $[b]+1$-times if $b$ is not an integer and $b$-times if $b$ is an integer) and Lemma 1.2, we obtain

$$
\begin{gathered}
\left(\int_{r}^{\infty} \frac{1}{t^{2 \sigma}} \int_{4 r<\left|x_{0}-u-y\right| \leq 2 t}\left|\frac{\Omega(x-u-y)}{|x-u-y|^{n-\rho}}-\frac{\Omega\left(x_{0}-u-y\right)}{\left|x_{0}-u-y\right|^{n-\rho}}\right|^{2}\right. \\
\left.\quad \times t^{2 n}\left(1+\log \frac{t}{r}\right)^{2 \gamma} \frac{d u d t}{t^{n+1}}\right)^{1 / 2} \\
\leq\left(\int_{4 r<\left|x_{0}-u-y\right|}\left|\frac{\Omega(x-u-y)}{|x-u-y|^{n-\rho}}-\frac{\Omega\left(x_{0}-u-y\right)}{\left|x_{0}-u-y\right|^{n-\rho}}\right|^{2}\right. \\
\left.\quad \times \int_{\left|x_{0}-u-y\right| / 2}^{\infty} \frac{\left(1+\log \frac{t}{r}\right)^{2 \gamma}}{t^{2 \sigma-n+1}} d t d u\right)^{1 / 2}
\end{gathered}
$$




$$
\begin{aligned}
& \leq\left(\int_{4 r<\left|x_{0}-u-y\right|}\left|\frac{\Omega(x-u-y)}{|x-u-y|^{n-\rho}}-\frac{\Omega\left(x_{0}-u-y\right)}{\left|x_{0}-u-y\right|^{n-\rho}}\right|^{2}\right. \\
& \left.\times \frac{\left(1+\log \frac{\left|x_{0}-u-y\right|}{r}\right)^{2 \gamma}}{\left|x_{0}-u-y\right|^{2 \sigma-n}} d u\right)^{1 / 2} \\
& \leq\left(\sum_{k=2}^{\infty} \int_{2^{k} r \leq\left|x_{0}-u-y\right|<2^{k+1} r}\left|\frac{\Omega(x-u-y)}{|x-u-y|^{n-\rho}}-\frac{\Omega\left(x_{0}-u-y\right)}{\left|x_{0}-u-y\right|^{n-\rho}}\right|^{2}\right. \\
& \left.\times \frac{\left(1+\log \frac{\left|x_{0}-u-y\right|}{r}\right)^{2 \gamma}}{\left|x_{0}-u-y\right|^{2 \sigma-n}} d u\right)^{1 / 2} \\
& \leq \sum_{k=2}^{\infty}\left(\int_{2^{k} r \leq\left|x_{0}-u-y\right|<2^{k+1} r}\left|\frac{\Omega(x-u-y)}{|x-u-y|^{n-\rho}}-\frac{\Omega\left(x_{0}-u-y\right)}{\left|x_{0}-u-y\right|^{n-\rho}}\right|^{2}\right. \\
& \left.\times \frac{\left(1+\log \frac{\left|x_{0}-u-y\right|}{f} r\right)^{2 \gamma}}{\left|x_{0}-u-y\right|^{2 \sigma-n}} d u\right)^{1 / 2} \\
& \leq C \sum_{k=2}^{\infty} \frac{(1+(k+1) \log 2)^{\gamma}}{\left(2^{k} r\right)^{\sigma-\frac{n}{2}}}\left(2^{k+1} r\right)^{\frac{n}{2}-(n-\sigma)} \\
& \times\left(\|\Omega\|_{L^{2}\left(S^{n-1}\right)} \frac{\left|x-x_{0}\right|}{2^{k} r}+\int_{\frac{\left|x-x_{0}\right|}{2^{k+1} r}}^{\frac{\left|x-x_{0}\right|}{2^{k} r}} \frac{\omega_{2}(\delta)}{\delta} d \delta\right) \\
& \leq C \sum_{k=2}^{\infty}\left(\|\Omega\|_{L^{2}\left(S^{n-1}\right)} \frac{(1+k)^{\gamma}}{2^{k}}+\frac{(1+k)^{\gamma}}{(1+k)^{\beta}} \int_{\frac{\left|x-x_{0}\right|}{2^{k+1} r}}^{\frac{\left|x-x_{0}\right|}{2^{k_{r}}}} \frac{\omega_{2}(\delta)}{\delta}(1+|\log \delta|)^{\beta} d \delta\right) \\
& \leq C\left(\|\Omega\|_{L^{2}\left(S^{n-1}\right)}+\int_{0}^{1} \frac{\omega_{2}(\delta)}{\delta}(1+|\log \delta|)^{\beta} d \delta\right) \text {. }
\end{aligned}
$$

Hence, we have

$$
\begin{aligned}
L_{31} & \leq C \int_{\mathbb{R}^{n}} \frac{\left|f_{3}(y)\right|}{\left|y-x_{0}\right|^{n} \log \gamma \frac{\left|y-x_{0}\right|}{r}} d y \\
& \leq C \sum_{k=2}^{\infty} \int_{2^{k} r \leq\left|y-x_{0}\right|<2^{k+1} r} \frac{\left|f(y)-f_{4 B}\right| d y}{\left|y-x_{0}\right|^{n} \log \gamma \frac{\left|y-x_{0}\right|}{r}} \\
& \leq C \sum_{k=2}^{\infty} \frac{1}{r^{n} 2^{n k} k^{\gamma}} \int_{\left|y-x_{0}\right|<2^{k+1}}\left|f(y)-f_{4 B}\right| d y \\
& \leq C \sum_{k=2}^{\infty} \frac{1}{r^{n} 2^{n k} k^{\gamma}}\|f\|_{\mathcal{E}^{\alpha, 1}} r^{\alpha}\left(2^{k+1} r\right)^{n} \leq C\left(\sum_{k=2}^{\infty} \frac{1}{k^{\gamma}}\right)\|f\|_{\mathcal{E}^{\alpha, p} r^{\alpha}} .
\end{aligned}
$$


In the fourth inequality in the above, we have used the inequality

$$
\left(\int_{\left|y-x_{0}\right| \leq R}\left|f_{3}(y)\right|^{p} d y\right)^{1 / p} \leq C\|f\|_{\mathcal{E}^{\alpha, p} r^{\alpha}} R^{n / p} .
$$

As for $L_{32}$, we have, in the same way as in the estimate for $L_{1}$,

$$
\begin{aligned}
& L_{32} \leq\left(\int_{r}^{\infty} \int_{\mathbb{R}^{n}}\left(\frac{1}{t^{\sigma}} \int_{\left|x_{0}-u-y\right| \leq 4 r} \frac{\left|\Omega\left(x_{0}-u-y\right) f_{3}(y)\right|}{\left|x_{0}-u-y\right|^{n-\sigma}} d y\right)^{2}\right. \\
& \left.\quad \times\left(\frac{t}{t+|u|}\right)^{2 n+2 \eta} \frac{d u d t}{t^{n+1}}\right)^{1 / 2} \\
& \leq \int_{\mathbb{R}^{n}}\left(\int_{r}^{\infty} \int_{\left|x_{0}-u-y\right| \leq 4 r} \frac{\left|\Omega\left(x_{0}-u-y\right)\right|^{2}}{\left|x_{0}-u-y\right|^{2 n-2 \sigma}}\left(\frac{t}{\left|y-x_{0}\right|}\right)^{2 n+2 \eta} \frac{d u d t}{t^{n+2 \sigma+1}}\right)^{1 / 2} \\
& \leq \int_{\mathbb{R}^{n}}\left(\int_{r}^{\infty} \int_{\left|x_{0}-u-y\right| \leq 4 r} \frac{\left|\Omega\left(x_{0}-u-y\right)\right|^{2}}{\left|x_{0}-u-y\right|^{2 n-2 \sigma}} \frac{d u d t}{t^{2 \sigma-n-2 \eta+1}}\right)^{1 / 2} \frac{\left|f_{3}(y)\right|}{\left|y-x_{0}\right|^{n+\eta}} d y \\
& \leq C\|\Omega\|_{L^{2}\left(S^{n-1}\right)} \int_{\mathbb{R}^{n}}\left(\int_{r}^{\infty} \int_{0}^{4 r} s^{2 \sigma-n-1} d s \frac{d t}{t^{2 \sigma-n-2 \eta+1}}\right)^{1 / 2} \frac{\left|f_{3}(y)\right|}{\left|y-x_{0}\right|^{n+\eta}} d y \\
& \leq C\|\Omega\|_{L^{2}\left(S^{n-1}\right)} r^{\eta}\|f\|_{\mathcal{E}^{\alpha, 1}} r^{\alpha-\eta} \leq C\|\Omega\|_{L^{2}\left(S^{n-1}\right)}\|f\|_{\mathcal{E}^{\alpha, p}, r^{\alpha}}
\end{aligned}
$$

Similarly we get the same estimate for $L_{33}$. Summing up the estimates for $L_{31}, L_{32}, L_{33}$, we obtain the desired estimate for $L_{3}$. Finally summing up the estimates for $L_{1}, L_{2}, L_{3}$, we get

$$
\left|\mu_{\lambda, \infty}^{*, \rho}\left(f_{3}\right)(x)-\mu_{\lambda, \infty}^{*, \rho}\left(f_{3}\right)\left(x_{0}\right)\right| \leq C\|f\|_{\mathcal{E}^{\alpha, p}} r^{\alpha} .
$$

Lemma 2.12. Let $\lambda>1,1 \leq p<\infty, 0<\alpha<\min \{1,(\lambda-1) n / 2+1 / 2\}$, and $\Omega \in L^{1}\left(S^{n-1}\right)$. Then there exists $C>0$ such that for any ball $B=$ $B\left(x_{0}, r\right)$ and any $f \in \mathcal{E}^{\alpha, p}$ satisfying $\mu_{\lambda, 0, \infty}^{*, \rho}\left(f_{3}\right)\left(x_{0}\right)<+\infty$, it holds

$$
\begin{gathered}
\mu_{\lambda, 0, \infty}^{*, \rho}\left(f_{3}\right)(x)<+\infty \quad \text { and } \\
\left|\mu_{\lambda, 0, \infty}^{*, \rho}\left(f_{3}\right)(x)-\mu_{\lambda, 0, \infty}^{*, \rho}\left(f_{3}\right)\left(x_{0}\right)\right| \leq C r^{\alpha}\|\Omega\|_{L^{1}\left(S^{n-1}\right)}\|f\|_{\mathcal{E}^{\alpha, p}}
\end{gathered}
$$

for any $x \in B$. 
Proof. For $x \in B$ we see easily

$$
\begin{aligned}
\mu_{\lambda, 0, \infty}^{*, \rho}\left(f_{3}\right)(x) \leq & \left(\int_{0}^{r} \int_{\left|u-x_{0}\right|>8 r}\left|\frac{1}{t^{\rho}} \int_{|y-u| \leq t} \frac{\Omega(u-y) f_{3}(y)}{|u-y|^{n-\rho}} d y\right|^{2}\right. \\
& \left.\times\left(1+\frac{|u-x|}{t}\right)^{-\lambda n} \frac{d u d t}{t^{n+1}}\right)^{1 / 2} \\
+ & \left(\int_{0}^{r} \int_{|u-x| \leq 9 r}\left|\frac{1}{t^{\rho}} \int_{|y-u| \leq t} \frac{\Omega(u-y) f_{3}(y)}{|u-y|^{n-\rho}} d y\right|^{2}\right. \\
& \left.\times\left(1+\frac{|u-x|}{t}\right)^{-\lambda n} \frac{d u d t}{t^{n+1}}\right)^{1 / 2} .
\end{aligned}
$$

We see by Lemma 2.10 (its variant replaced $8 r$ by $9 r$ ) that the second term in the right-hand side of the above inequality is bounded by $C^{\alpha}\|\Omega\|_{L^{1}\left(S^{n-1}\right)}$ $\|f\|_{\mathcal{E}^{\alpha, p}}$. Hence, we have

$$
\begin{array}{rl}
\mu_{\lambda, 0, \infty}^{*, \rho}\left(f_{3}\right)(x) \leq & C r^{\alpha}\|\Omega\|_{L^{1}\left(S^{n-1}\right)}\|f\|_{\mathcal{E}^{\alpha, p}}+\mu_{\lambda, 0, \infty}^{*, \rho}\left(f_{3}\right)\left(x_{0}\right) \\
& +\left(\int_{0}^{r} \int_{\left|u-x_{0}\right|>8 r}\left|\frac{1}{t^{\rho}} \int_{|y-u| \leq t} \frac{\Omega(u-y) f_{3}(y)}{|u-y|^{n-\rho}} d y\right|^{2}\right. \\
\left.\quad \times\left|\left(1+\frac{|u-x|}{t}\right)^{-\lambda n}-\left(1+\frac{\left|u-x_{0}\right|}{t}\right)^{-\lambda n}\right| \frac{d u d t}{t^{n+1}}\right)^{1 / 2} & C r^{\alpha}\|\Omega\|_{L^{1}\left(S^{n-1}\right)}\|f\|_{\mathcal{E}^{\alpha, p}}+\mu_{\lambda, 0, \infty}^{*, \rho}\left(f_{3}\right)\left(x_{0}\right)+I, \text { say. }
\end{array}
$$

As in the proof of Lemma 2.9 (c), we have for every $0<t<r$ and $u \in \mathbb{R}^{n}$ with $\left|u-x_{0}\right|>8 r$

$$
\begin{aligned}
\left|\int_{|y-u| \leq t} \frac{\Omega(u-y) f_{3}(y)}{|u-y|^{n-\rho}} d y\right| & \leq C\|\Omega\|_{L^{1}\left(S^{n-1}\right)}\|f\|_{\operatorname{Lip}_{\alpha}}\left(t+\left|u-x_{0}\right|\right)^{\alpha} t^{\sigma} \\
& \leq C\|\Omega\|_{L^{1}\left(S^{n-1}\right)}\|f\|_{\operatorname{Lip}_{\alpha}}\left|u-x_{0}\right|^{\alpha} t^{\sigma}
\end{aligned}
$$

By the mean value theorem, we get

$$
\begin{aligned}
& \left|\left(1+\frac{|u-x|}{t}\right)^{-\lambda n}-\left(1+\frac{\left|u-x_{0}\right|}{t}\right)^{-\lambda n}\right| \\
& \leq C \frac{\left|x-x_{0}\right|}{t}\left(1+\frac{\left|u-x_{0}\right|}{t}\right)^{-\lambda n-1} \leq C \frac{\left|x-x_{0}\right| t^{\lambda n}}{\left|u-x_{0}\right|^{\lambda n+1}}
\end{aligned}
$$


Hence, we have

$$
\begin{aligned}
I \leq C\left(\int_{0}^{r} \int_{\left|u-x_{0}\right|>8 r}\left(\frac{1}{t^{\sigma}}\|\Omega\|_{L^{1}\left(S^{n-1}\right)}\|f\|_{\operatorname{Lip}_{\alpha}}\left|u-x_{0}\right|^{\alpha} t^{\sigma}\right)^{2}\right. \\
\left.\quad \times \frac{\left|x-x_{0}\right| t^{\lambda n}}{\left|u-x_{0}\right|} \frac{d u d t}{t^{n+1}}\right)^{1 / 2} \\
\leq C\|\Omega\|_{L^{1}\left(S^{n-1}\right)}\|f\|_{\operatorname{Lip}_{\alpha}} r^{1 / 2} \\
\quad \times\left(\int_{0}^{r}\left(\int_{\left|u-x_{0}\right|>8 r}\left|u-x_{0}\right|^{2 \alpha-\lambda n-1} d u\right) t^{\lambda n-n-1} d t\right)^{1 / 2} \\
\leq C\|\Omega\|_{L^{1}\left(S^{n-1}\right)}\|f\|_{\operatorname{Lip}_{\alpha}} r^{\alpha} .
\end{aligned}
$$

Thus, we have

$$
\mu_{\lambda, 0, \infty}^{*, \rho}\left(f_{3}\right)(x) \leq \mu_{\lambda, 0, \infty}^{*, \rho}\left(f_{3}\right)\left(x_{0}\right)+C r^{\alpha}\|\Omega\|_{L^{1}\left(S^{n-1}\right)}\|f\|_{\mathcal{E}^{\alpha, p}} \text { for any } x \in B .
$$

Reversing the roles of $\mu_{\lambda, 0, \infty}^{*, \rho}\left(f_{3}\right)\left(x_{0}\right)$ and $\mu_{\lambda, 0, \infty}^{*, \rho}\left(f_{3}\right)(x)$, we have

$$
\mu_{\lambda, 0, \infty}^{*, \rho}\left(f_{3}\right)\left(x_{0}\right) \leq \mu_{\lambda, 0, \infty}^{*, \rho}\left(f_{3}\right)(x)+C r^{\alpha}\|\Omega\|_{L^{1}\left(S^{n-1}\right)}\|f\|_{\mathcal{E}^{\alpha, p}} \text { for any } x \in B
$$

and hence we have

$$
\left|\mu_{\lambda, 0, \infty}^{*, \rho}\left(f_{3}\right)(x)-\mu_{\lambda, 0, \infty}^{*, \rho}\left(f_{3}\right)\left(x_{0}\right)\right| \leq C r^{\alpha}\|\Omega\|_{L^{1}\left(S^{n-1}\right)}\|f\|_{\mathcal{E}^{\alpha, p}} \text { for any } x \in B \text {. }
$$

\section{§3. Proofs of Theorems 1-9}

Now, we will prove the theorems.

Proof of Theorem 1. We follow the ideas by Kurtz [12] and Sun [20]. Let $r>0$ and $B=B\left(x_{0}, r\right)$. Set $f_{1}=f_{4 B}, f_{2}=\left(f-f_{4 B}\right) \chi_{4 B}$ and $f_{3}=\left(f-f_{4 B}\right) \chi_{(4 B)^{c}}$. Then, $f=f_{1}+f_{2}+f_{3}$ and $\mu^{\rho}\left(f_{1}\right)=0$.

By assumption, $\mu^{\rho}(f)\left(x_{0}\right)<\infty$. So, we have $\mu_{\infty}^{\rho}(f)\left(x_{0}\right) \leq \mu^{\rho}(f)\left(x_{0}\right)<$ $\infty$. Using Lemma 2.1 we have $\mu_{\infty}^{\rho}\left(f_{3}\right)\left(x_{0}\right) \leq \mu_{\infty}^{\rho}(f)\left(x_{0}\right)+\mu_{\infty}^{\rho}\left(f_{2}\right)\left(x_{0}\right)<\infty$. Hence by Lemmas 2.2 and 2.3 (i) we have for $x \in B$

$$
\mu^{\rho}\left(f_{3}\right)(x) \leq \mu_{0}^{\rho}\left(f_{3}\right)(x)+\mu_{\infty}^{\rho}\left(f_{3}\right)(x) \leq C r^{\alpha}\|f\|_{\mathrm{BMO}}+\mu_{\infty}^{\rho}\left(f_{3}\right)\left(x_{0}\right)<\infty
$$

and

$$
\left|\mu^{\rho}\left(f_{3}\right)(x)-\mu^{\rho}\left(f_{3}\right)\left(x_{0}\right)\right|=\left|\mu_{\infty}^{\rho}\left(f_{3}\right)(x)-\mu_{\infty}^{\rho}\left(f_{3}\right)\left(x_{0}\right)\right| \leq C\|f\|_{\mathrm{BMO}}
$$


Using $L^{2}$-boundedness of $\mu^{\rho}$ (Theorem B) we have $\left\|\mu^{\rho}\left(f_{2}\right)\right\|_{L^{2}} \leq C\left\|f_{2}\right\|_{L^{2}}$, and from this it follows that $\mu^{\rho}\left(f_{2}\right)(x)<\infty$ for almost all $x \in B$. Thus, we have $\mu^{\rho}(f)(x) \leq \mu^{\rho}\left(f_{2}\right)(x)+\mu^{\rho}\left(f_{3}\right)(x)<\infty$ for almost all $x \in B$. Since $r$ is arbitrary, we see that $\mu^{\rho}(f)(x)<\infty$ for almost all $x \in \mathbb{R}^{n}$.

Let $E=\left\{x \in \mathbb{R}^{n} ; \mu^{\rho}(f)(x)<\infty\right\}$. We have only to show that for any ball $B=B\left(x_{0}, r\right)$ with center $x_{0} \in E$,

$$
\int_{B}\left|\mu^{\rho}(f)(x)-\left(\mu^{\rho}(f)\right)_{B}\right| d x \leq C|B|\|f\|_{\mathrm{BMO}}
$$

Set $f=f_{1}+f_{2}+f_{3}$ as above. Noting $\mu^{\rho}\left(f_{1}\right)=0$, and using $\left\|\mu^{\rho}\left(f_{2}\right)\right\|_{L^{2}} \leq$ $C\left\|f_{2}\right\|_{L^{2}} \leq C|B|^{1 / 2}\|f\|_{\mathcal{E}^{0,2}} \leq C|B|^{1 / 2}\|f\|_{\text {BMO }}$ and the above inequality for $\mu^{\rho}\left(f_{3}\right)$, we have

$$
\begin{aligned}
& \frac{1}{|B|} \int_{B}\left|\mu^{\rho}(f)(x)-\left(\mu^{\rho}(f)\right)_{B}\right| d x \leq \frac{2}{|B|} \int_{B}\left|\mu^{\rho}(f)(x)-\mu^{\rho}\left(f_{3}\right)\left(x_{0}\right)\right| d x \\
& \quad=\frac{2}{|B|} \int_{B}\left|\mu^{\rho}\left(f_{2}+f_{3}\right)(x)-\mu^{\rho}\left(f_{3}\right)(x)+\mu^{\rho}\left(f_{3}\right)(x)-\mu^{\rho}\left(f_{3}\right)\left(x_{0}\right)\right| d x \\
& \leq \frac{2}{|B|} \int_{B}\left|\mu^{\rho}\left(f_{2}\right)(x)\right| d x+\frac{2}{|B|} \int_{B}\left|\mu^{\rho}\left(f_{3}\right)(x)-\mu^{\rho}\left(f_{3}\right)\left(x_{0}\right)\right| d x \\
& \leq C\left(\frac{1}{|B|} \int_{4 B}\left|f_{2}(x)\right|^{2} d x\right)^{1 / 2}+C\|f\|_{\mathcal{E}^{0,1}} \leq C\|f\|_{\text {BMO }}
\end{aligned}
$$

This completes the proof of Theorem 1.

Proof of Theorem 2. The proof is the same as that of Theorem 1. We use Lemmas 2.1, 2.2, 2.3 (ii) and Theorem B. We note that, since $\Omega$ satisfies $L^{1}-\beta$-Dini condition, it satisfies $L^{1}$-Dini condition and hence $\Omega \in$ $L \log ^{+} L\left(S^{n-1}\right) \varsubsetneqq H^{1}\left(S^{n-1}\right)$. So, we can use Theorem B.

Proof of Theorem 3. The proof is the same as that of Theorem 1. We use Lemmas 2.1, 2.2, 2.3 (iii), and Theorem B.

Proof of Theorem 4. The proof is the same as that of Theorem 1. We use $\mu_{S, 0}^{\rho}$ and $\mu_{S, \infty}^{\rho}$, Lemmas 2.4, 2.5, 2.6 (i), and Theorem A (ii) $(p=2)$.

Proof of Theorem 5. The proof is the same as that of Theorem 1. We use $\mu_{S, 0}^{\rho}$ and $\mu_{S, \infty}^{\rho}$, Lemmas 2.4, 2.5, 2.6 (ii), (iii) and Theorem A (ii) $(p=2)$. In the case $1 / 2 \leq \alpha<1$, as is noted in the proof of Theorem 2 , we see $\Omega \in L \log ^{+} L\left(S^{n-1}\right)$, and we can apply Theorem A (ii). 
Proof of Theorem 6. (i) Since $\mathcal{E}^{\alpha, p}=L^{p}\left(\mathbb{R}^{n}\right)$ for $\alpha=n / p$, by Theorem A we have only to treat the case $n / p<\alpha<0$. So, the proof is the same as that of Theorem 1. We use $\mu_{S, 0}^{\rho}$ and $\mu_{S, \infty}^{\rho}$, Lemmas 2.4, 2.5, 2.6 (iv) and Theorem A.

(ii) The proof is the same as the above case (i). We use 2.6 (v) in place of 2.6 (iv). Note that if $1<p<2$, the condition $\sigma>n / 2$ implies $\frac{2 n}{n+2 \sigma}<1$, and so we can apply Theorem A.

Proof of Theorem 7. The proof is the same as that of Theorem 1. We use $\mu_{\lambda, 0}^{*, \rho}$ and $\mu_{\lambda, \infty}^{*, \rho}$, Lemmas 2.7, $2.9(\mathrm{~b}), 2.11$ (i), and Theorem A (ii) $(p=2)$.

Proof of Theorem 8. We follow the proof of Theorem 1 by modifying it. Let $r>0$ and $B=B\left(x_{0}, r\right)$. Set $f_{1}=f_{4 B}, f_{2}=\left(f-f_{4 B}\right) \chi_{4 B}$ and $f_{3}=\left(f-f_{4 B}\right) \chi_{(4 B)^{c}}$. Then, $f=f_{1}+f_{2}+f_{3}$ and $\mu_{\lambda}^{*, \rho}\left(f_{1}\right)=0$.

(i) The case $0<\alpha<1 / 2$. By assumption, $\mu_{\lambda}^{*, \rho}(f)\left(x_{0}\right)<\infty$. So, we have $\mu_{\lambda, \infty}^{*, \rho}(f)\left(x_{0}\right)+\mu_{\lambda, 0, \infty}^{*, \rho}(f)\left(x_{0}\right) \leq 2 \mu_{\lambda}^{*, \rho}(f)\left(x_{0}\right)<\infty$. Using Lemmas 2.7 and 2.8 we have $\mu_{\lambda, \infty}^{*, \rho}\left(f_{3}\right)\left(x_{0}\right)+\mu_{\lambda, 0, \infty}^{*, \rho}\left(f_{3}\right)\left(x_{0}\right) \leq \mu_{\lambda, \infty}^{*, \rho}(f)\left(x_{0}\right)+$ $\mu_{\lambda, 0, \infty}^{*, \rho}(f)\left(x_{0}\right)+\mu_{\lambda, \infty}^{*, \rho}\left(f_{2}\right)\left(x_{0}\right)+\mu_{\lambda, 0, \infty}^{*, \rho}\left(f_{2}\right)\left(x_{0}\right)<\infty$. Hence by Lemmas 2.10, 2.11 and 2.12 we have for $x \in B$

$$
\begin{aligned}
\mu_{\lambda}^{*, \rho}\left(f_{3}\right)(x) & \leq \mu_{\lambda, 0,0}^{*, \rho}\left(f_{3}\right)(x)+\mu_{\lambda, 0, \infty}^{*, \rho}\left(f_{3}\right)(x)+\mu_{\lambda, \infty}^{*, \rho}\left(f_{3}\right)(x) \\
& \leq 3 C r^{\alpha}\|f\|_{\mathcal{E}^{\alpha, p}}+\mu_{\lambda, 0, \infty}^{*, \rho}\left(f_{3}\right)\left(x_{0}\right)+\mu_{\lambda, \infty}^{*, \rho}\left(f_{3}\right)\left(x_{0}\right)<\infty,
\end{aligned}
$$

and

$$
\begin{aligned}
& \left|\mu_{\lambda}^{*, \rho}\left(f_{3}\right)(x)-\mu_{\lambda}^{*, \rho}\left(f_{3}\right)\left(x_{0}\right)\right| \leq\left|\mu_{\lambda, 0,0}^{*, \rho}\left(f_{3}\right)(x)-\mu_{\lambda, 0,0}^{*, \rho}\left(f_{3}\right)\left(x_{0}\right)\right| \\
& \quad+\left|\mu_{\lambda, 0, \infty}^{*, \rho}\left(f_{3}\right)(x)-\mu_{\lambda, 0, \infty}^{*, \rho}\left(f_{3}\right)\left(x_{0}\right)\right|+\left|\mu_{\lambda, \infty}^{*, \rho}\left(f_{3}\right)(x)-\mu_{\lambda, \infty}^{*, \rho}\left(f_{3}\right)\left(x_{0}\right)\right| \\
& \quad \leq 4 C r^{\alpha}\|f\|_{\mathcal{E}^{\alpha, p}}
\end{aligned}
$$

Using $L^{p}$-boundedness of $\mu_{\lambda}^{*, \rho}$ (Theorem A) we have $\left\|\mu_{\lambda}^{*, \rho}\left(f_{2}\right)\right\|_{L^{p}} \leq$ $C\left\|f_{2}\right\|_{L^{p}}$, and from this it follows that $\mu_{\lambda}^{*, \rho}\left(f_{2}\right)(x)<\infty$ for almost all $x \in B$. Thus, we have $\mu_{\lambda}^{*, \rho}(f)(x) \leq \mu_{\lambda}^{*, \rho}\left(f_{2}\right)(x)+\mu_{\lambda}^{*, \rho}\left(f_{3}\right)(x)<\infty$ for almost all $x \in B$. Since $r$ is arbitrary, we see that $\mu_{\lambda}^{*, \rho}(f)(x)<\infty$ for almost all $x \in \mathbb{R}^{n}$.

The rest of the proof is the same as that of Theorem 1. We omit it.

(ii) The case $1 / 2 \leq \alpha<1$. In this case, the proof is simpler than the case (i), and the same as that of Theorem 1 . We use $\mu_{\lambda, 0}^{*, \rho}$ and $\mu_{\lambda, \infty}^{*, \rho}$, 
Lemmas 2.7, 2.9 and 2.11 (iii). As is noted in the proof of Theorem 5, we see $\Omega \in L \log ^{+} L\left(S^{n-1}\right)$, and we can apply Theorem A (ii). This completes the proof of Theorem 8 .

Proof of Theorem 9. (i) The proof is the same as that of Theorem 6 (i). We use $\mu_{\lambda, 0}^{*, \rho}$ and $\mu_{\lambda, \infty}^{*, \rho}$, Lemmas 2.7, 2.9, 2.11 (iv), and Theorem A.

(ii) The proof is the same as the above case (i). We use 2.11 (v) in place of 2.11 (iv). Note that if $1<p<2$, the condition $\sigma>n / 2$ implies $\frac{2 n}{n+2 \sigma}<1$, and so we can apply Theorem A.

Acknowledgments. The authors would like to express their deep thanks to the referee for his/her very careful reading and many valuable comments and suggestions.

\section{REFERENCES}

[1] A. P. Calderón, M. Weiss and A. Zygmund, On the existence of singular integrals, Proc. Sympos. Pure Math., 10 (1967), 56-73.

[2] L. Colzani, Hardy spaces on Spheres, Ph.D thesis, Washington University, St. Louis, MO, 1982.

[3] L. Colzani, M. Taibleson and G. Weiss, Maximal estimates for Cesàro and Riesz means on spheres, Indiana Univ. Math. J., 33 (1984), 873-889.

[4] Y. Ding, D. Fan and Y. Pan, $L^{p}$-boundedness of Marcinkiewicz integrals with Hardy space function kernel, Acta. Math. Sinica (English series), 16 (2000), 593-600.

[5] Y. Ding, S. Lu and Q. Xue, On Marcinkiewicz integral with homogeneous kernels, J. Math. Anal. Appl., 245 (2000), 471-488.

[6] Y. Ding, S. Lu and K. Yabuta, A problem on rough parametric Marcinkiewicz functions, J. Austral. Math. Soc., 72 (2002), 13-21.

[7] Y. Ding and Q. Xue, Weak type $(1,1)$ bounds for a class of the Littlewood-Paley operators, J. Math. Soc. Japan, 57 (2005), 184-194.

[8] Y. Ding, Q. Xue and K. Yabuta, Weighted estimate for a class of Littlewood-Paley operators, to appear in Taiwanese J. Math.

[9] E. B. Fabes, R. L. Johnson and U. Neri, Spaces of harmonic functions representable by Poisson integrals of functions in BMO and $\mathcal{L}_{p, \lambda}$, Indiana Univ. Math. J., 25 (1976), 159-170.

[10] Y. Han, On some properties of s-function and Marcinkiewicz integrals, Acta Sci. Natur. Univ. Pekinensis, 5 (1987), 21-34.

[11] L. Hörmander, Translation invariant operators, Acta Math., 104 (1960), 93-139.

[12] D. S. Kurtz, Littlewood-Paley operators on BMO, Proc. Amer. Math. Soc., 99 (1987), 657-666.

[13] D. S. Kurtz and R. L. Wheeden, Results on weighted norm inequalities for multipliers, Trans. Amer. Math. Soc., 255 (1979), 343-362. 
[14] J. Peetre, On the theory of $\mathcal{L}_{p, \lambda}$ spaces, J. Funct. Anal., 4 (1969), 71-87.

[15] S. Qiu, Boundedness of Littlewood-Paley operators and Marcinkiewicz integral on $\mathcal{E}^{\alpha, p}$, J. Math. Res. Exposition, 12 (1992), 41-50.

[16] M. Sakamoto and K. Yabuta, Boundedness of Marcinkiewicz functions, Studia Math., 135 (1999), 103-142.

[17] E. M. Stein, On the functions of Littlewood-Paley, Lusin, and Marcinkiewicz, Trans. Amer. Math. Soc., 88 (1958), 430-466.

[18] E. M. Stein, Singular integrals and differentiability properties of functions, Princeton Univ. Press, Princeton, N.J., 1970.

[19] E. M. Stein and G. Weiss, Introduction to Fourier Analysis on Euclidean Spaces, Princeton Univ. Press, Princeton, N.J., 1971.

[20] Y. Sun, On the existence and boundedness of square function operators on Campanato spaces, Nagoya Math. J., 173 (2004), 139-151.

[21] A. Torchinsky, Real-Variable Methods in Harmonic Analysis, Academic Press, San Diego, Calif., 1986.

[22] A. Torchinsky and S. Wang, A note on the Marcinkiewicz integral, Colloq. Math., 60/61 (1990), 235-243.

[23] S. Wang, Boundedness of the Littlewood-Paley g-function on $\operatorname{Lip}_{\alpha}\left(\mathbb{R}^{n}\right)(0<\alpha<1)$, Illinois J. Math., 33 (1989), 531-541.

[24] S. Wang, Some properties of the Littlewood-Paley g-function, Contemp. Math., 42 (1985), 191-202.

[25] S. Wang and J. Chen, Some notes on square function operator, Annals of Mathematics (Chinese), Series A, 11 (1990), 630-638.

[26] Q. Xue, Parametrized Littlewood-Paley operators, Thesis, Beijing Normal University, 2004.

[27] K. Yabuta, Boundedness of Littlewood-Paley operators, Math. Japonica, 43 (1996), 143-150.

[28] K. Yabuta, Some remarks to Marcinkiewicz functions, Kwansei Gakuin Univ. Nat. Sci. Rev., 6 (2002), 9-15.

[29] K. Yabuta, Existence and boundedness of $g_{\lambda}^{*}$-function and Marcinkiewicz functions on Campanato spaces, Sci. Math. Jpn., 59 (2004), 93-112.

Yong Ding

Department of Mathematics

Beijing Normal University

Beijing, 100875

P. R. of China

dingy@bnu. edu.cn 
Qingying Xue

Department of Mathematics

Beijing Normal University

Beijing, 100875

P. R. of China

xqyyan@sohu.com

Current address:

School of Science and Technology

Kwansei Gakuin University

Gakuen 2-1

Sanda, Hyogo 669-1337

Japan

qyxue@ksc.kwansei.ac.jp

Kôzô Yabuta

School of Science and Technology

Kwansei Gakuin University

Gakuen 2-1

Sanda, Hyogo 669-1337

Japan

yabuta@ksc.kwansei.ac.jp 